\title{
A REVIEW OF OPTICAL AND RADIATIVE PROPERTIES OF NANOPARTICLE SUSPENSIONS: EFFECTS OF PARTICLE STABILITY, AGGLOMERATION, AND SEDIMENTATION
}

\author{
Layth Al-Gebory ${ }^{1,2, *} \mathcal{E}$ M. Pinar Mengüiç ${ }^{1}$ \\ ${ }^{1}$ Department of Mechanical Engineering and CEEE, Özyeğin University, \\ Çekmeköy, 34794 Istanbul, Turkey \\ ${ }^{2}$ Department of Materials Engineering, University of Technology, Baghdad, Iraq
}

*Address all correspondence to: Layth Al-Gebory, Department of Mechanical Engineering and CEEE, Özyeğin University, Çekmeköy, 34794 Istanbul, Turkey; Tel.: +90 507822 2956; Fax: +90 216564 9999, E-mail: layth.ismael@ozu.edu.tr; 130006@uotechnology.edu.iq

Original Manuscript Submitted: 12/29/2019; Final Draft Received: 2/3/2020

Nanoparticle suspensions (NPSs) are suspensions of nanosize particles in base fluids in the form of a solid-liquid mixture. They are used extensively in a wide range of industrial and engineering applications, and show considerably different and tunable, physiochemical, thermal, and radiative properties compared to other solutions. It has been demonstrated that added nanoparticles can significantly alter and enhance the optical and radiative properties of the base fluids. There is still a concern about the particle agglomeration and sedimentation behaviors and the long-term stability of NPSs under different conditions, which may limit their potential reliable applications. There is a close relationship between the particle agglomeration and the optical and radiative properties of nanoparticle suspensions. In this review, the relationship between these fundamental properties with the emphasis on the procedural stability of nanoparticle suspensions is explored. Different issues related to the preparation and the characterization of NPSs, their sedimentation, the effects of particle types, sizes on individual, hybrid, and agglomerated nanoparticle suspensions, as well as the effect of $\mathrm{pH}$ values, are discussed. Their effects on the optical and spectral radiative properties are also discussed. Simplified analyses are outlined based on dependent-independent scattering demarcation and effective properties of NPSs.

KEY WORDS: nanofluids, stability, agglomeration, optical properties, radiative properties, dependent scattering, independent scattering, heat transfer enhancement

\section{INTRODUCTION}

With the advances in technology and with the ever-increasing demand to miniaturize devices, there is a need to develop new paradigms for thermal management of different types of equipment and systems. Cooling load for such systems increases exponentially, necessitating the new types of working media as cooling fluids. During the past two decades, many common base 
fluids modified by suspending nanosize solid particles in them for this purpose (Devendiran and Amirtham, 2016; Kumar et al., 2019). These new-generation fluids are called nanoparticle suspensions (NPSs) or nanofluids (NFs) (Choi et al., 2004). NPSs can be prepared by dispersing single-type NPs in a base fluid (mono-NPSs) or by dispersing more than one type of NPs in a base fluid (hybrid NPSs). Since the first synthesis of NPSs, they have shown the potential to alter the thermophysical and optical/radiative properties compared with those of base fluids. This alteration can be manifested to significant enhancement of the properties of NPSs, which contributes to improving the behavior and efficiency of many different systems, including solar thermal power plant and solar chemical processes (Wang et al., 2003; Keblinski et al., 2005; Khanafer and Vafai, 2018). Although NPSs may enhance the transfer of mechanical and thermal energy, they have some drawbacks as well. For example, they may have poor dispersibility, and lose their stability because of particle agglomeration and rapid sedimentation (Karimzadehkhouei et al., 2016). In addition, due to the adhering of particles to the inner surfaces of the system, their heat transfer performance can degrade, which may cause an increase in the pumping power, and may even cause the blockage of small pipes (Wen et al., 2009; Raja et al., 2016).

In NPSs, particle concentration and system heterogeneity have important influences on the heat and mass transfer, where the thermal transport in NPSs depends on different parameters such as particle size and size distribution, shape, particle concentration, and sedimentation (Nayak and Mishra, 2019). Therefore, it is quite important to include the effects of all these parameters in the investigations in order to explore their impacts on the mechanisms of heat and mass transfer in different types of NPSs (Choi et al., 2004; Xie et al., 2005; Asadi et al., 2018; Yousif et al., 2019).

One of the major challenges NPSs face is the preparation and long-term stability, which are the principal prerequisites for achieving reliable optical and radiative properties. NPSs go through an unavoidable settling motion due to gravity, which can be overcome by induced Brownian motion and enhancing the repulsive force between particles to prevent their collisions. Thus, as long as the particles remain unagglomerated, or if agglomerate sizes can be controlled, it is possible to have stable NPSs (Daungthongsuk and Wongwises, 2007; Devendiran and Amirtham, 2016).

Homogeneous mixtures are mainly composed of gases and miscible liquids, e.g., air or a mixture of water and alcohol. The composition of a homogeneous mixture is two or more substances that may not be noticeable in the mixture, even when using a microscope. Also, the constituents of a homogeneous mixture are not easily separated by using mechanical means such as precipitation or centrifugation. A heterogeneous mixture is composed of parts that can be distinguished with the naked eye or by using a microscope (Gibbs, 1879; Dai and Haussener, 2018). Basically, everything except the vacuum is heterogeneous. Even in media that are considered to be homogeneous (e.g., pure gases and liquids), it is possible to distinguish the individual heterogeneities (molecules and atoms) with specific methods. However, for most practical purposes, air and water are considered homogeneous. Colloidal suspensions, emulsions, and NPSs are composed of distinguishable parts and are naturally heterogeneous mixtures. Yet, NPSs can be characterized as homogeneous suspensions. This term implies that the solid particles are uniformly distributed in the fluid, and the suspension has the characteristics of a homogeneous mixture. Homogeneity and heterogeneity of particulate systems have important effects on the optical and radiative properties in addition to their effect on the radiative transfer.

Nanoparticles, which are smaller than $100 \mathrm{~nm}$ or so and uniformly distributed in a solution, absorb and scatter the incident radiative energy. Their contribution to scattering increases with 
increasing particle sizes, which is usually the result of agglomeration or sedimentation process. Absorption and scattering by particles can be quantified by solving the electromagnetic wave (Maxwell) equations. The EM-wave analysis provides a complete solution for absorption and scattering cross sections of individual particles if the particle size, structure, and optical properties (complex index of refraction) are available (Bohren and Huffman, 2008; Mishchenko, 2014; Howell et al., 2015). If particles are far from each other, then a linear summation of the absorption and scattering cross sections of different size, shape particles, and agglomerates can be used (Aslan et al., 2006b). However, if the particles are within close proximity of each other or if they are touching, then these simple additions of individual properties would not be accurate. The reason for this is simple: when the interaction of a spherical particle with an EM-wave is considered (as in the case of Lorenz-Mie formulation), it is assumed that a plane wave is incident on a particle. If particles are close to each other, this assumption would not be valid as the EM wave incident on the particles cannot be considered plane wave anymore. Under these conditions, the interactions of particles with the incoming light (EM-waves) should either be modeled rigorously (to account for the waves coming from a large number of scatterers) or approximated. A Discrete Dipole Approximation, T-matrix approach, or another rigorous solution model can be used to rigorously model the absorption and scattering by these complex structures (Mishchenko and Dlugach, 2018, 2019).

However, these solution schemes are usually very time-consuming and may not be readily used for engineering processes. To circumvent this computational complexity, in the 1980s a dependent scattering approach was proposed by Drolen (Drolen and Tien, 1987) and his students (REFS). In these systems, the behavior of a complex agglomerate is related to the absorption and scattering of individual particles by a form factor. Such a form factor depends on the number of individual particles, their size, and the proximity of them to each other.

Over the last twenty years, the research on NPSs has been rapidly increasing due to the importance of their unique properties and the range of applicability (Afrand et al., 2017; Xiao et al., 2017; Guo, 2019). This growing trend for nanosuspensions is depicted in Fig. 1, which is based on a number of publications retrieved from "Web of Science." This figure shows that research publications related to NPSs are growing so rapidly that the total number of publications in 2018 is $30 \%$ of the entire number of relevant papers published over the past twenty years. In this analysis, the keyword "nanoparticle suspensions" includes the words nanoparticle suspensions, nanofluids, and nanosuspensions. The keyword "optical/radiative properties" is also included in the search, to find the papers related to radiative transfer, radiative properties, electromagnetic radiation, scattering, absorption, emission, and optical properties.

This chart shows that research regarding NPSs is growing so rapidly that the total number of publications in 2018 is $30 \%$ of the entire number of relevant papers published over the past twenty years. In this figure, the search of the keywords has been done in the title of the published papers. If the search was relaxed to the full text of published papers, it is becoming increasingly more challenging to provide a comprehensive review of all the types of NPSs because of the vast amounts of related literature. For that reason, in the last two years a few papers have reviewed the literature in a focused fashion and on certain types of NPSs (Yang and Du, 2017).

The present paper provides a detailed review of optical and radiative properties of nanoparticle suspensions with a focus on the preparation, stability, and characterization of nanoparticle suspensions and their impacts on the optical and radiative properties. The effects of different parameters regarding nanoparticle stability, agglomeration, and sedimentation are discussed. The effective scattering simplifications are presented, where different theoretical and experimental methods are proposed for describing the optical and radiative transfer of NPSs. The radiative

Volume 27, Issue 3, 2020 


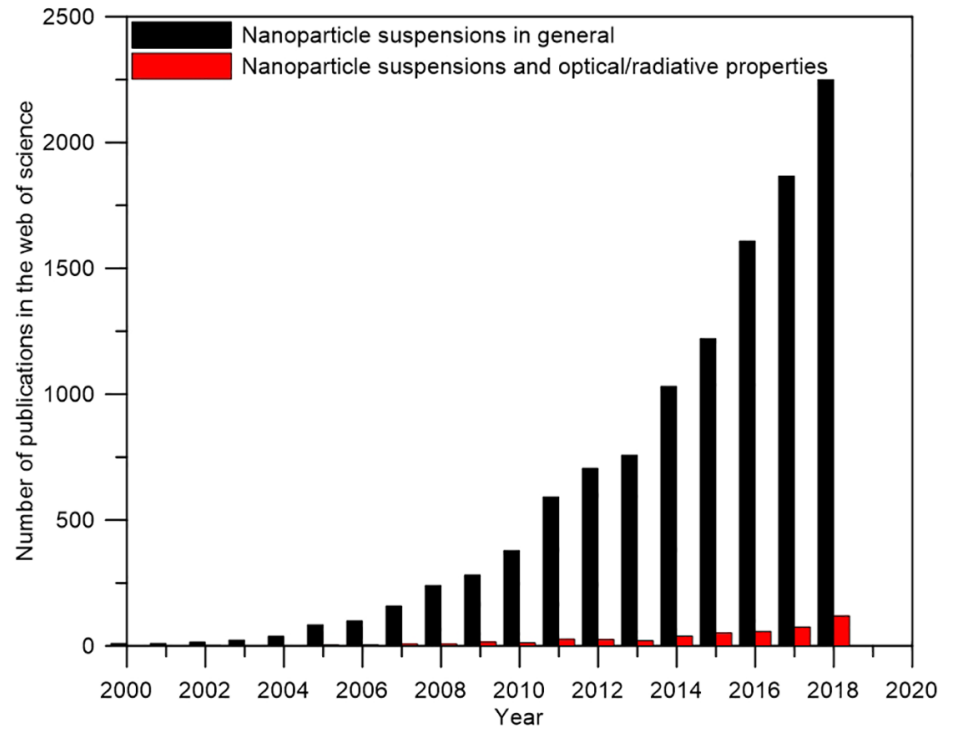

FIG. 1: Number of publications containing "nanoparticle suspensions" in the title retrieved from the web of science

properties of the different types of NPSs under different conditions are summarized and discussed, although the fundamental solution techniques based on the Maxwell equations are not included. Instead, a simplified discussion on dependent-independent scattering is provided and their boundaries of different regimes are discussed for practical applications. Finally, the radiative heat transfer applications involving NPSs are outlined.

\section{STABILITY OF NANOPARTICLE SUSPENSIONS}

\subsection{Introduction}

Nanoparticles may exist in many configurations that range from a well-dispersed mode to a totally agglomerated state (Chen et al., 2004; Das et al., 2018). A considerable number of investigations have been carried out on the stability of NPSs over the last few decades, including their preparation and characterization for different applications (Trisaksri and Wongwises, 2007; Ali et al., 2018). The stability of NPSs is the main issue for both scientific research and for practical applications. To maintain the stability of NPSs, the two different approaches are commonly used, including electrostatic stabilization or the addition of surfactants to a given suspension. In hybrid nanoparticles, the stability of the suspended particles remains a major challenge because such suspensions involve two or more different types of nanoparticles. Usually, the stability is measured after the preparation of the selected NPSs; yet the change in their stabilities is hardly measured after the experiments conducted under different conditions (Devendiran and Amirtham, 2016).

There are several practical issues and challenges regarding nanoparticle agglomeration that need to be addressed and overcome before this field of study can be fully established. The main issues can be listed as: (i) The disagreements between most of the experimental data and the theoretical model predictions should be understood and quantified; (ii) The poor characterization 
of the NPSs should be overcome and the new measurement and characterization methodologies should be developed; and (iii) The lack of understanding of the complex physical phenomena responsible for the anomalous behavior of NPSs should be avoided by using new techniques.

The NPSs stability is classified into three categories: (i) Kinetic stability: Nanoparticles that are dispersed in the NPSs due to the Brownian motion, overcoming their sedimentation caused by gravitational force; (ii) Dispersion stability: Because of the agglomeration of the nanoparticles, their dispersion into the fluids may deteriorate over time; and (iii) Chemical stability: Chemical reactions between the suspended nanoparticles or between the base fluid and nanoparticles are to be avoided. Thus, it is a necessary requirement to carefully prepare the NPSs to ensure their long-term stability following these three criteria (Das et al., 2007; Kiruba et al., 2018). There are several practical issues and challenges regarding nanoparticle agglomeration that need to be addressed and overcome before this field of study can be fully established. The main issues can be listed as: (i) The disagreements between most of the experimental data and the theoretical model predictions should be understood and quantified; (ii) The poor characterization of the NPSs should be overcome and the new measurement and characterization methodologies should be developed; and (iii) The lack of understanding of the complex physical phenomena responsible for the anomalous behavior of NPSs should be avoided by using new techniques.

\subsection{Preparation of Nanoparticle Suspensions}

Preparing NPSs presents many challenges, both technical and financial. The main technical difficulty is in the production of a homogeneous suspension of the nanoparticles, mostly because the particles always tend to agglomerate due to the strong van der Waals interactions (Wang and Mujumdar, 2008; Azizi et al., 2018). The most important step in the preparation of a NPS is the mixing of the nanoparticles with the base fluid. The delicate preparation of a NPS is important because NPSs need special requirements such as an even suspension, stable suspension, low agglomeration of particles, and no chemical change in the fluid (Lee et al., 1999; Aziz, 2018).

A number of methods have been discussed regarding the preparation of NPSs, which can be divided into two approaches (one-step and two-step approaches) (Aslan et al., 2006b; Hwang et al., 2008). For one-step approaches, the NPSs are synthesized by dispersing the NPs directly in the base fluids. The vaporization of the base materials under vacuum conditions is also considered. One positive regarding this approach is that the agglomeration of the nanoparticles is minimized. However, the negative is that the vapor pressure of the fluid is required to be very low. Because the NPSs are prepared in situ, only small quantities can be produced and this is mostly done in batches. This precludes the mass production of the NPSs in industrial processes that can be commercially available (Liu et al., 2005; Asadi et al., 2018).

In the two-step approach, the synthesized nanoparticles with the desired shape and size are dispersed in the base fluids. The important advantage of this approach is that NPs can be produced separately using different types of NPs and the medium, which allows their cheap and huge production. The main disadvantage of this approach is that the particles agglomerate in the base fluids. Producing a long-term stable suspension using the two-step method remains a challenge. However, this method is more preferable when the long-term stability of the nanoparticles can be achieved by reducing the agglomeration rate (Yang et al., 2017).

Xuan and Li recommended methods to be used for the stabilization of the suspensions: (i) changing the $\mathrm{pH}$ value of suspension, (ii) using surface activators and/or dispersants, and (iii)

Volume 27, Issue 3, 2020 
using ultrasonic vibration. These different methods can alter the surface properties of the suspended particles and may be used to suppress the formation of particle clusters in order to attain stable suspensions. Using these techniques is dependent on the necessary application of the NPSs. The choice of suitable activators and dispersants depends mainly upon the properties of both the solutions and particles. The stability of the dispersion of the nanoparticle in the base fluid is shown by the zeta potential value, and a high zeta potential value indicates good stability (Xuan and Li, 2000; Dalkilic et al., 2018).

Over the years, a significant amount of studies have addressed the problem of the solidfluid interaction phenomenon (Kawanami and Sakurai, 2007; Choi et al., 2018). However, the impact of different factors such as the particle shape and size, particle agglomeration and size distribution, and particle stabilization on the properties and performance of NPSs have yet to be extensively investigated. The probability of particle agglomeration increases with the decreasing particle size especially at the nanosize, i.e., for particles less than $100 \mathrm{~nm}$. A stable NPS means that the suspended particle size does not increase significantly due to agglomeration (Du and Tang, 2015; Almohammed and Breuer, 2016).

The mechanisms for suspension of the nanoparticles in base fluids have also been investigated using different methods to produce long-term stable nanosuspensions and to display a clear idea about suspended particle mechanisms (Boisvert et al., 2001; Widegren and Bergström, 2002). The dispersion of $\mathrm{Al}_{2} \mathrm{O}_{3}$ and $\mathrm{TiO}_{2}$ nanoparticles in the base fluids have received wide interest in recent years (Fazio et al., 2008; Safaei-Naeini et al., 2012; Asadi et al., 2018). There have been studies in regard to the suspensions of $\mathrm{TiO}_{2}$ and $\mathrm{Al}_{2} \mathrm{O}_{3}$ nanoparticles in different aqueous or non-aqueous media using different mechanisms of dispersions, and there have also been studies on the stability of the prepared suspensions using different methods of stability enhancement (Boisvert et al., 2001; Saltiel et al., 2004). Aqueous $\mathrm{TiO}_{2} /$ water NPSs in differing particle sizes and concentrations displayed stable behavior for months after the preparation without any dispersants (He et al., 2007; Muthusamy et al., 2016). The investigations were carried out on the $\mathrm{Al}_{2} \mathrm{O}_{3}$ /water NPSs (particle size of $13 \mathrm{~nm}$ and with particle concentration of $0.05-0.1 \%$ vol.) and showed that good stability was achieved (Said et al., 2013). Additionally, the stability of different NPSs $\left(\mathrm{Al}_{2} \mathrm{O}_{3}, \mathrm{ZnO}\right.$, and $\mathrm{Fe}_{2} \mathrm{O}_{3} /$ water) with the particle sizes $(45,60$, and $30 \mathrm{~nm}$, respectively) were investigated without using any additives, which showed that the $\mathrm{Al}_{2} \mathrm{O}_{3}$ /water NPSs had better stability than the others (Colangelo et al., 2013).

A cavitating flow through micro-orifices was proposed to enhance the stability of water $/ \gamma$ $\mathrm{Al}_{2} \mathrm{O}_{3}$ NPSs to prevent particle agglomeration and sedimentation. In this method, the particle agglomerates interact with the emerging bubbles and lead to a decrease in the agglomerates size through the generated shock waves by the bubbles collapsing. No surfactants or surface modifiers were used in the preparation of the nanofluids (Karimzadehkhouei et al., 2016). An experimental investigation was performed on the nucleate pool boiling heat transfer of water$\mathrm{TiO}_{2}$ and $\mathrm{CuO}$ nanoparticles-based nanofluids. The results show that the performance of the heat transfer was enhanced when the nanoparticles were placed in water. The enhancement was extremely dependent on the concentration of the particles. For the water- $\mathrm{TiO}_{2}$ nanofluid, the largest enhancement was approximately $15 \%$ for the particle mass fraction of $0.001 \%$. However, for the water-CuO nanofluid, the enhancement was more than $35 \%$ for the particle mass fraction of $0.2 \%$ (Karimzadehkhouei et al., 2017).

A homogeneous NPS with a single type of nanoparticle may not necessarily include all the required characteristics for thermal, optical, and rheological requirements. Then, NPSs can be prepared using different types of nanoparticles to tune the required properties for a specific application in hand. These hybrid nanofluids are new types of nanofluids and can be prepared 
by suspending two or more nanoparticles in the base fluid, that is to say, hybrid (composite) nanoparticles in the base fluid. A hybrid nanoparticle is a substance combined with the physical and chemical properties of the constituent materials simultaneously and gives these properties in a homogeneous phase. However, the behavior of hybrid NPSs in a working environment has yet to be studied extensively. Some hybrid NPSs are expected to yield better thermophysical and radiative properties when compared with individual NPSs, and they offer a potential for thermal applications, including solar thermal systems or other applications that require desired and tunable radiative properties (Wang et al., 2003; Keblinski and Cahill, 2005; Ito et al., 2018). In addition, when compared to individual nanofluids due to the synergistic effect, the hybrid nanofluid is expected to yield better thermal conductivity compared to single NPSs (Sarkar et al., 2015; Sinz et al., 2016).

The main aim of hybrid nanofluid research is to assure its application in any equipment connected to heat transfer. A detailed experimental study to determine the physicochemical properties of hybrid nanostructures for biotechnology application was conducted by Turco et al. (2007) and Kiruba et al. (2018). In a study similar to the aforementioned, a hybrid nano-polymer was prepared for the applications in the solar cells as well for applications in evacuated tube solar collectors (Jamil et al., 2016; Muhammad et al., 2016; Sundar et al., 2017). Moreover, the use of $\mathrm{Al}_{2} \mathrm{O}_{3}-\mathrm{Cu}$ /water hybrid nanofluid in heat sink for an application in cooling electronics has demonstrated an increase in convective heat transfer compared to water (Selvakumar and Suresh, 2012). Alumina-silver nanocomposite hybrid nanofluid in a helical heat exchanger to examine the thermal performance and pressure drop was used in another study. In this study, the hybrid nanofluid showed a higher percentage of heat transfer $(31.6 \%)$ in comparison to the conventional heat transfer fluid (water) (Allahyar et al., 2016). Contrary to this, different experiments of $\mathrm{Cu} / \mathrm{TiO}_{2}$ hybrid nanocomposite/water in a tubular heat exchanger showed an increase in the overall heat transfer of $30.4 \%$ at a volume concentration of $0.7 \%$ (Madhesh and Kalaiselvam, 2014).

Generally, surfactants are required in the preparation of carbon nanotube (CNTs) solutions to obtain stable NPSs (Kozan et al., 2008; Hordy et al., 2014; Choi et al., 2018). Additionally, the investigation showed that the stability of graphite/water NPSs could be achieved by using polyvinylpyrrolidone (PVP) surfactant (Lee et al., 2007). The investigation on the multi-walled carbon nanotubes (MWCNTs) with the particle size range of 10-30 nm and concentration of 0.2 wt.\% in water showed unstable NPSs samples without surfactants (Yousefi et al., 2012). Due to the agglomeration of the single-walled carbon nanotubes (SWCNTs) in the distilled water, a considerable increase in the transmittance was observed after some minutes of preparation because of their sedimentation. SWCNTs have diameter $=1.2-1.5 \mathrm{~nm}$ and length $2-5 \mu \mathrm{m}$, and the CNT concentration was 5-100 mg/l (Gorji et al., 2015).

\subsection{Characterization of Nanoparticle Suspensions}

Different theoretical methods and experimental techniques are used for the particle characterization of NPSs, including earlier studies (Aslan et al., 2003; Saltiel et al., 2004; Kozan and Mengüç, 2008). Theoretical methods may be too slow to determine the behavior of a real NPSs system where a large number of particle agglomerates occur (the real particulate suspensions). Advanced measurement systems and optimization algorithms are needed to infer a meaningful interpretation of the results using the theoretical methods (Aslan et al., 2006b; Jing and Song, 2017). Understanding the changes in the number of particles of a particular size is important to understand the agglomeration phenomenon in the NPSs. The characterization of the NPSs

Volume 27, Issue 3, 2020 
always reveals several important details such as the nanoparticle shape, size, distribution, and stability of the nanoparticles in the base fluid. A general theory has been developed in the past for agglomeration, which is referred to as the Derjaguin-Landau-Verwey-Overbeek theory, or the DLVO theory (Derjaguin, 1941; Verwey, 1947). Accordingly, the agglomeration process is modeled by the combination of the attractive and repulsive forces that act on a pair of particles in a single potential energy function.

The agglomeration of the nanoparticles in the NPSs is characterized by many microscale behaviors. The investigation of particle agglomeration can be divided into two methods: direct detection (Song et al., 2016; Chen et al., 2017) and indirect feedback (Fedele et al., 2011; Anushree and Philip, 2016). The meaning of direct detection is that the evolution of particle size distribution (PSD) in time is directly determined by Dynamic Light Scattering (DLS), electrical microscope (SEM and TEM), or other method types. In this way, one can obtain instant information about the nanoparticle agglomeration in NPSs (Saltiel et al., 2005). Usually, the difficulty in its operation is the disadvantage of direct detection. First, much sample quantity is required. Some DLS instruments need dozens of samples of milliliters and several measurements are necessary. However, if the one-step method is used to prepare the sample, the sample quantity will be extremely limited. Second, for some concentrated suspensions, the process of agglomeration is very rapid, which may affect the robustness of experiments. While a long time (in minutes) is often required for each measurement, the direct detection method cannot really capture this dynamic process (Song et al., 2016; Chen et al., 2017). Indirect feedback is to acquire the agglomeration process by testing the changing of the thermophysical and property, the optical property or stability (concentration distribution) of the suspension. The advantage of this method is its ease of operation, while the disadvantage is that it cannot obtain quantitative PSD, but only the qualitative dispersed state of nanoparticles in the nanofluid. In the practical application, direct detection and indirect feedback are usually combined due to their complementary properties (Fedele et al., 2011; Anushree and Philip, 2016).

A comparative study of several measurement techniques used for the characterization of dispersions was conducted by Mahl et al. (2011). They investigated dispersions with almost monodisperse spherical silver nanoparticles $(\sim 70 \mathrm{~nm})$, and gold nanoparticles $(\sim 15 \mathrm{~nm})$ and these dispersions were characterized using differing techniques: scanning electron microscopy (SEM), transmission electron microscopy (TEM), dynamic light scattering (DLS), and analytical disc centrifugation (ADC). This is also denoted as differential centrifugal sedimentation (DCS), and nanoparticle tracking analysis (NTA). The limits of the measurement techniques were examined as the different techniques could generate comparable results with respect to particle size as the main task. In addition, how the size distribution data for the mixture when compared to those of the individual particles was investigated (Mahl et al., 2011). Different methods of nanomaterial characterization and proposed dynamic light scattering (DLS) as a useful technique to evaluate particle size, size distribution, and the zeta potential of nanomaterials in solution was reviewed by Powers et al. (2006).

DLS was used in both recent, and past studies going back to 1975 as a base method for analyzing the suspension stability and measurement of the particle size in solution (Berne and Pecora, 2000; Saltiel et al., 2004; Wu et al., 2005; Williams et al., 2006; Simakov and Tsur, 2007). Detailed experimental/theoretical studies were conducted by the Mengüç group in order to explore the core nature of individual cotton fibers via light-scattering experiments (Aslan et al., 2003). A new precision nephelometer was built and calibrated with quartz fiber for this purpose. The key parameters for the precision measurements determined were the opening (viewing angle), and scanning range and rate. A comparison of the experimental results was made regarding 
the theoretical predictions based on the finite-element model. It was determined that the scattered intensity profiles as a function of the scattering angle may be connected to the quality (fineness) of the cotton (Aslan et al., 2003).

In a later study, they measured the size and structure of the colloidal metal oxide $(\mathrm{MgBaFeO})$ particles using an Elliptically Polarized Light Scattering (EPLS) technique (Aslan et al., 2006b). This approach was based on a hybrid experimental/theoretical study where the experimental data were compared to predictions attained using a T-Matrix model, which accounts for the irregularities of the particle shape. A power-law distribution function with two parameters was employed to account for the size of the particle distribution (Aslan et al., 2006b).

Table 1 shows the sizes of particle agglomerates for $\mathrm{Al}_{2} \mathrm{O}_{3} \mathrm{NP}$ as obtained from TEM, DLS, and DLVO theory. The sedimentation rate is also shown in this table (Sajid et al., 2014). Compared with the samples prepared in distilled water only, the distribution of the nanoparticles used in these experiments was not very fine.

\section{4 pH Value and Agglomeration in Nanoparticle Suspensions}

Most of the particles in nature are found in agglomerated forms, and understanding particle agglomeration concepts and mechanisms is important in a wide range of applications and processes. There are different factors that cause and affect the interaction (attractive) force between particles which is one of the factors whereby the particles form bonds. Particle agglomeration behavior is controlled by surface forces; attractive and repulsive forces. With the decreasing particle size, particularly particles at the nanosize, the probability of particle agglomeration increases. That is because the distance between the particles decreases with the decreasing particle size making the attractive force more important, where the extremely large surface area of particles is in comparison with their volume (Mewis and Wagner, 2012; Tadros, 2012; Das et al., 2018).

The $\mathrm{pH}$ value of particulate suspensions is one of the keys to achieve the stability of NPSs; $\mathrm{pH}$ value refers to the hydrogen ions (positive and negative ions). For good stability, the $\mathrm{pH}$ value of the NPSs need to be adjusted to a value far from the isoelectric point $\left(\mathrm{pH}_{i s o}\right.$, the point at which the particles have low zeta potential) for nanoparticles, where a large value of the zeta potential is obtained. The structure and size of the nanoparticles and agglomerates are dependent on the different parameters, related to both the particles' composition and the boundary conditions. Nanoparticles in base fluids usually agglomerate, and one of the most significant challenges in the NPSs research field is to produce well-dispersed and long-term stable (low particle agglomeration ratio) NPSs. The stability of the NPSs is impacted upon by different parameters such as the $\mathrm{pH}$ level of the suspension, particle size and shape, surfactant concentration, and sonication time (Wamkam et al., 2011; Konakanchi et al., 2015).

TABLE 1: Nanoparticles agglomeration of water-based $\mathrm{Al}_{2} \mathrm{O}_{3}\left(d_{p}=13 \mathrm{~nm}\right)$, $(\emptyset=0.05 \%)$ at $25^{\circ} \mathrm{C}$ (see Sajid et al., 2014 for more details)

\begin{tabular}{|c|c|c|c|c|}
\hline \multirow{2}{*}{$\boldsymbol{t}(\mathbf{h})$} & \multicolumn{4}{|c|}{ Average diameter of agglomerates (nm) } \\
\cline { 2 - 5 } & TEM & DLVO Theory & DLS & Sedimentation rate (m/s) \\
\hline 2 & 20.2 & 19.5 & 137 & $3.86 \mathrm{E}-10$ \\
\hline 4 & 28.3 & 19.5 & 137 & $4.55 \mathrm{E}-10$ \\
\hline 240 & 130 & 126 & 137 & $4.06 \mathrm{E}-09$ \\
\hline
\end{tabular}

Volume 27, Issue 3, 2020 
As the particle agglomeration process continues, large clusters form. The growth, in general, takes place through encounters between the different clusters, and thus one refers to the clustercluster agglomeration process. The resulting clusters are not regular but are statistically selfsimilar. One refers to the diffusion-limited cluster agglomeration (DLCA) or reaction-limited cluster agglomeration (RLCA) depending on whether the agglomeration is fast or slow. The clusters have various features in each regime. DLCA clusters are loose and ramified, but the RLCA clusters are more compact. The distribution of the cluster size also varies in these two regimes. The $\mathrm{pH}$ value carries a significant impact on the stability behavior of the nanoparticles, which in turn carries the dominant impact on the particle agglomeration rate (Prasher et al., 2006; Ali et al., 2018). Differing sizes and structures (linear, porous, and compact) of particle agglomerates are produced dependent on the $\mathrm{pH}$ value of the particulate suspensions. The larger the cluster size is, the quicker their settling velocity is, which in turn causes particle sedimentation (Russel et al., 1989; Hunter, 2001; Khanafer and Vafai, 2018).

An increase or decrease of potential energy means that the forces between the particles are repulsive or attractive, respectively. In a suspension, the drastic and ceaseless Brownian motion may cause the nanoparticles to collide. These collisions reduce the distance between the nanoparticles; therefore, the van der Waals attraction would hold them together without separating them again. This process of agglomeration is known as DLCA (Lattuada et al., 2003; Cosgrove, 2010). However, in the RLCA, only if the collision happens and the particles react with each other will the agglomerates be formed. The difference between them is that in DLCA, the particles will form an agglomerate if collision occurs (Lattuada et al., 2003). It is clear that the pH of NPSs produces different types of particle agglomerates and shows different trends in the optical and radiative properties in addition to the scattering behavior (dependent and independent) (Al-Gebory and Mengüç, 2018).

To prevent the re-agglomeration in the $\mathrm{TiO}_{2}$ nanosuspensions, the $\mathrm{pH}$ value can be adjusted to 11 to achieve a higher zeta potential $(40 \mathrm{mV})$ (He et al., 2007). $\mathrm{Al}_{2} \mathrm{O}_{3}-\mathrm{H}_{2} \mathrm{O}$ nanofluids were synthesized; their dispersion behaviors and thermal conductivity in water were examined under various $\mathrm{pH}$ values and sodium dodecylbenzene sulfonate (SDBS) concentrations. The results showed the stability and thermal conductivity enhancements of $\mathrm{Al}_{2} \mathrm{O}_{3}-\mathrm{H}_{2} \mathrm{O}$ nanofluids are extremely dependent on the $\mathrm{pH}$ values and differing SDBS dispersant concentrations of nanosuspensions (Zhu et al., 2009). In order to aim at the dispersion stability of nanofluids, Xian-Ju et al. scrutinized the absorbency and the zeta potential of $\mathrm{TiO}_{2}$ and $\mathrm{Al}_{2} \mathrm{O}_{3}$ nanofluids under various $\mathrm{pH}$ values and dispersant concentrations. The results determined that in the mass fraction $0.05 \%$ alumina and $0.01 \%$ titanium dioxide nanosuspensions, the absolute value of the zeta potential and the absorbency of the two nanofluids with sodium dodecyl sulfate (SDS) dispersant are their highest at an optimal $\mathrm{pH}\left(\mathrm{Al}_{2} \mathrm{O}_{3} \approx 6.0, \mathrm{TiO}_{2} \approx 9.5\right)$. In addition, it was found that there was a good correlation between the absorbency and zeta potential: the higher the absolute value of the zeta potential was, the greater the absorbency was, and the better the stability of the system was (Xian-Ju et al., 2011). The suspension of the $0.1 \%$ wt. of the $\mathrm{TiO}_{2}$ nanoparticles into the water was performed with the dispersion methods including adding SDS, and by adjusting the $\mathrm{pH}$ value to 5 by using the ultrasonic vibration technique, it was found that the nanoparticles can remain stable for over one year (Ghadimi and Metselaar, 2013). Viscosity, heat transfer, and pressure loss measurements were performed for $\mathrm{TiO}_{2}$ nanofluids with a nominal particle size of $27 \mathrm{~nm}$ with a concentration of up to 3 vol. $\%$ at $\mathrm{pH}=10$, and single-phase correlations were found to be successfully extended to such nanosuspensions (Vakili et al., 2013).

The investigation demonstrated that the stability of the $\mathrm{TiO}_{2} /$ water nanosuspensions with the particle size of $25 \mathrm{~nm}$ and with the particle concentration of $20-50 \mathrm{wt}$. \% at $\mathrm{pH}=11$ without 
any dispersant was stable for more than one month after the preparation. The ultrasonic vibration path was also used in the preparation (Sen et al., 2015). The stable $\mathrm{TiO}_{2}$ nanosuspensions were obtained successfully from the surface-modified nanoparticles in the same medium, where the particle concentration was up to $50 \%$ wt., and with low viscosity, the highest particle concentration (20\% wt.) was achieved for the $\mathrm{TiO}_{2}$ nanoparticles with an average particle size of $25 \mathrm{~nm}$ in aqueous solutions at $\mathrm{pH}=11$, where these suspensions were limited by particle agglomeration and high viscosity (Sen et al., 2015). This method enhances the stability of the nanoparticles by achieving a higher zeta potential to avoid the adhesion of the nanoparticles by the higher electrostatic repulsion barriers (Devendiran and Amirtham, 2016). To achieve good dispersion for the NPSs, the adjustment of the $\mathrm{pH}$ value has been adopted by several researchers (Yang and Du, 2017).

Figure 2 shows the agglomeration time constant $\left(t_{p}\right)$ for different radii of alumina nanoparticles and indicates that $t_{p}$ decreases significantly with decreasing nanoparticle size $\left(r_{p}\right)$. The reason for this behavior is because, at the same volume fraction, smaller particles are closer together than larger particles, which leads to a higher attraction due to van der Waals forces. Figure 2 also shows $t_{p}$ for $\mathrm{pH}=5.0$ and $\mathrm{pH}=9.1$ (the isoelectric point for alumina). At $\mathrm{pH}=$ $9.1, t_{p}$ is very small because the alumina at this $\mathrm{pH}$, making the repulsive energy zero. Figure 2 also shows that $t_{p}$ decreases with increasing temperature because increasing temperature leads to a higher probability for particles to agglomeration (Prasher et al., 2006; Almohammed and Breuer, 2016).

\section{OPTICAL AND RADIATIVE PROPERTIES OF NANOPARTICLE SUSPENSIONS}

\subsection{Introduction}

Light is an electromagnetic wave. We are familiar with both electricity and magnetism, and we mostly view them as separate phenomena. For example, touching a metal object after walking

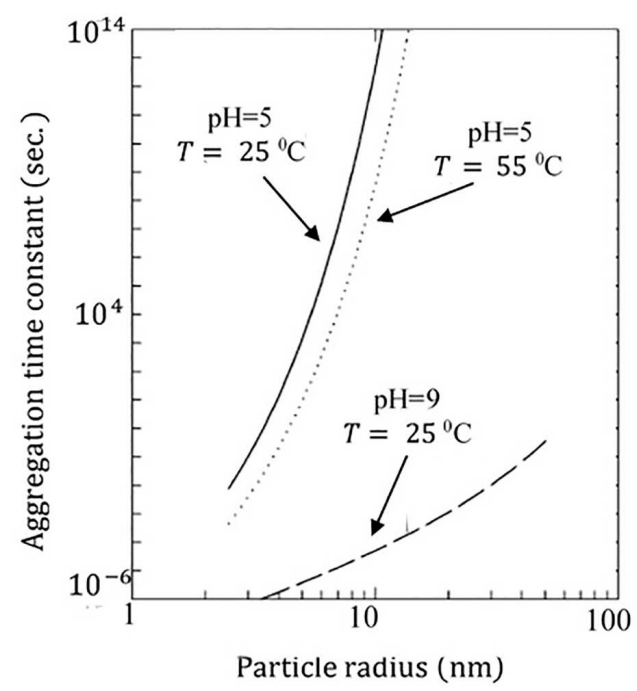

FIG. 2: Temperature and $\mathrm{pH}$ dependent $\mathrm{Al}_{2} \mathrm{O}_{3}$ nanoparticles agglomeration (DLVO theory) (see Prasher et al., 2006 for more details)

Volume 27, Issue 3, 2020 
across a carpet in the winter will lead to an electrical shock, and never to a magnetic shock. Similarly, our credit and debit cards carry magnetic, not electrical, strips. That said, in the mid-1800s Maxwell showed that these two phenomena are in fact intimately related and one cannot exist without the other (Maxwell, 1864; Mishchenko and Dlugach, 2018). It is light's nature to travel unhindered through a uniform medium. However, when it crosses an interface from a material with one refractive index into a second material with a different refractive index, this travel is interrupted, and light responds to this change in refractive index (RI) in three fundamentally different ways. Specifically, light can reflect, refract, and/or diffract at the interface of the two materials (Mishchenko, 2014; Howell et al., 2015).

In many systems relevant to high-temperature energy technologies, thermal radiation is a dominant mode of energy transfer. The majority of systems within this category are either porous in nature or contain particulates that actively participate in radiative transfer processes. Various examples of the particulate systems are fluidized and packed beds, fluidized bed and surface combustors, catalytic reactors, microsphere and fibrous insulations, ceramic foam insulations, ceramic and metallic screens, porous and sintered materials, particulate solar collectors, and liquid-droplet radiators, which are used in space applications (Viskanta and Mengüç, 1989; Dombrovsky and Baillis, 2010; Dombrovsky et al., 2018). Other examples where radiation from particles plays a significant role are a nuclear winter scenario, large-scale fires, and radiation transfer in the core debris of a melted reactor (Mengüç and Viskanta, 1986) or in a containment building and numerous others (Gupta et al., 2012; Dhand et al., 2015).

Radiative transfers as well as combined heat transfer by conduction and radiation through participating, dispersed media capable of absorbing, emitting, and scattering radiation are significant problems in terms of engineering (Viskanta and Mengüç, 1989; Howell et al., 2015). All media scatter light and when photons' so-called electromagnetic waves interact with a medium including small particles, the radiative intensity may be changed due to scattering and/or absorption (Mengüç, 2003). Direction of scattering an electromagnetic wave by a particle depends on important parameters, which include the shape and the type of the particle, the clearance between particles, its relative size, medium properties, and incident wavelength (Bohren and Huffman, 2008; Mishchenko, 2017).

The radiative transfer has a crucial role in various engineering applications that comprise continuous solid or fluid media embedding dispersed particles, which are generally referred to as "dispersed media" (Viskanta and Mengüç, 1989; Dombrovsky and Baillis, 2010; Mishchenko and Dlugach, 2018). Examples of these applications are the photo-thermal energy conversion systems, the solar-thermochemical energy conversion, material processing, microstructure diagnostic via light scattering, fire suppression by water sprays, thermal insulations, and thermal barrier coating, etc. If a substance (solid, liquid, or gas) is so finely insolubly distributed in a homogeneous or heterogeneous medium that it is in a considerably higher state of energy, by virtue of a large surface area, then in its compact phase the system will be considered as the dispersed phase. In this definition, there are materials which contain holes dispersed in a solid matrix, where the scattering of radiation with bubbles instead of particles is to be considered.

High porosity materials, such as fibers, powders, foams, refractory materials, porous solids, composite materials, fluidized, and packed beds are widely used in many industrial applications. In such materials, the relative importance of different modes of heat transfer depends on the structure and the physical properties, as well as on the operating conditions. From a radiative transfer perspective, these materials act as participating media. For an accurate prediction of the coupled heat transfer, a simultaneous solution of the energy conservation equation and of the radiative transfer equation (RTE) is required. Of course, also needed are the radiative properties 
of the medium (Modest, 2013; Howell et al., 2015; Hoffman and Driggers, 2016). One obvious reason for this is the rapid increasing progress in modeling radiative heat transfer in two- and three-dimensional, radioactively participating dispersed media.

There are, however, many additional problems related to radiative transfer that have yet to be analyzed. In particular, radiative heat transfer in heterogeneous ones or multidimensional dispersed media are not well understood. Considering the importance of such systems to practical and industrial applications, the need for more research in this area has been recognized (Viskanta and Mengüç, 1989; Dombrovsky and Baillis, 2010). Because an exact solution for the radiative transfer issue in these particular systems cannot be achieved, at least in the near future, coarse approximations are required. Treating the dispersed medium as continuous and homogeneous, and using the standard RTE with "effective radiative properties," which is different from the radiative properties of dilute media, is the most common assumption. This approximate method is known as a "homogeneous phase approach, HPA." Another less-frequent technique is called the "multiphase approach, MPA," consisting of assigning to both continuous and dispersed phases their own transport equation, but coupled with each other and their own effective radiative properties. Therefore, through the MPA, each phase is able to have its own temperature field (Randrianalisoa and Baillis, 2010; Saghrouni et al., 2019).

\subsection{Electromagnetic Wave Interaction with Particles and Agglomerates}

When electromagnetic radiations interact with particulate media, which include different particle agglomerates, the intensity of light along the direction of propagation may change due to scattering and/or absorption. Particle agglomeration has a significant effect on the radiative transfer and the optical and radiative properties of the NPSs. In most studies, it is assumed that a planar wave is incident on a particle, being a spherical shape or in agglomerated form. Absorption and scattering of EM waves by particles and agglomerates can be modeled by solving the electromagnetic-wave and particle interactions. Rigorous solutions of the Maxwell equations for spherical particles, cylinders, and many other irregular shapes are available in the literature (Mackowski and Mishchenko, 1996; Bohren and Huffman, 2008; Mishchenko and Dlugach, 2018). Also, numerical methods such as Discrete Dipole Approximation (Draine and Flatau, 1994; Yurkin and Hoekstra, 2007) and T-matrix approximation can be used (Mishchenko et al., 1996).

Using DDA, orientation and location of nanosize particles in agglomerates can be accounted for in the analysis in a rigorous fashion (Flatau and Draine, 2012). However, if a simpler LorenzMie solution is to be used for all particles in a cloud assuming they are spherical in shape, then their relative proximity with each other needs to be considered (Bohren and Huffman, 2008; Mishchenko, 2014; Howell et al., 2015). If a scattered wave is incident on a second particle near the first one, then the wave is not planar anymore unless the distance between these particles is more than roughly three wavelengths or more. This effect, although it can be taken care with the use of a rigorous EM-wave theory approach (Mishchenko et al., 2002; Mishchenko, 2014), is usually too cumbersome to be considered in the process industry. Instead, dependent/independent scattering regimes can be used to help for the design and development of robust process sensors.

It has been found that nanoparticle suspensions are effective electromagnetic wave absorbers within the UV-visible range (Gorji and Ranjbar, 2017). Consequently, the radiative properties of the suspended particles and the media containing those particles need to be investigated intensely. As there are many particles in a particulate medium, the scattered photon from a particle

Volume 27, Issue 3, 2020 
may interact with those from other particles. This means incident beams can be scattered more than one time and they can be scattered again by the nearby particles (Mishchenko et al., 1999; Dai and Haussener, 2018; Doicu and Mishchenko, 2018; Mishchenko and Dlugach, 2018).

In the past, several experimental studies were conducted by our group for single scattering measurements using different polarization settings of the incident scattered light (Mengüç and Manickavasagam, 1998; Mengüç, 2003; Saltiel et al., 2005). The effect of polarization allowed a finer resolution in detecting different size particles. Ivezic and Mengüç explored this behavior to determine the onset of soot agglomeration process and proposed a correlation to use if the particles are closer to each other (Ivezić and Mengüç, 1996; Ivezić et al., 1997). This correlation was derived in a different fashion than those proposed in earlier studies by Drolen and his group (Drolen and Tien, 1987).

When the diameter of the scattering particle is similar to the wavelength of light, scattering is dominated by diffraction. An unusual feature of this important interaction is that the scattering size of the particle can be larger than its physical size; that is, diffraction can occur even if the light ray does not directly strike the particle but instead passes a short distance from it. In general, scattering is the strongest when there is a large difference in refractive indices and the particle size and wavelength of light are similar to one another. Light can be considered completely scattered when it has been scattered enough times that its direction is completely randomized (this condition is referred to as "diffuse scattering"). It is tempting to extend this logic and conclude that decreasing the size of particles will always increase light-scattering intensity per unit weight. Up to a point, this is correct. However, for reasons discussed later, we find that there is a lower limit to particle size for efficient light scattering. Reflection is the redirection of light to the mirror angle of its incidence on the interface, while refraction is the deflection of that portion of the light entering the new medium from its initial direction. When a particle of a refractive index is embedded in a second material of another refractive index, the balance of these three phenomena (reflection, refraction, and diffraction) is determined to a great extent by the size of the particle compared to the wavelength of the light. Three broad size regimes exist: For large (macroscopic) objects, so-called geometric interactions occur between light and the object. When the particle dimensions and light wavelength are roughly comparable, light interacts via Mie scattering. For particles much smaller than the wavelength of light, light scatters through a mechanism first described by Rayleigh (Modest, 2013; Howell et al., 2015).

The scattering matrix element for agglomerates with different fractals was demonstrated. The influences of the different parameters such as the number and size of the monomers in agglomerates, the fractal dimension, as well as the wavelength of the incident light, were considered (Klusek et al., 2003). The small-angle light-scattering technique was also used to determine the morphology of the different particle agglomerates by considering the fractal theory (Kozan and Mengüç, 2008). A procedure was discussed to find the agglomeration of soot particles in flames from multi-wavelength experiments. $N$-particle agglomerates are modeled by using a discrete dipole approximation. It was discovered that it is possible that the measurements of the differential scattering and absorption coefficients, and phase function, can be used for the determination of the number of agglomerated monomers, their size, and the structure of the formed agglomerate. The representative results were presented for a complex index of the refraction value typical for those soot particles that are produced during the combustion of hydrocarbon fuels (Ivezić et al., 1997). By using the discrete dipole approximation (DDA), the light scattering from irregularly shaped particles with three different kinds of agglomerate morphologies was computed. It was discovered that even though there were noticeable differences in the particle morphology, their light-scattering responses were very similar, and the difference in light 
scattering very rarely was in accession of the error bars that occur in the laboratory measurements of micron-sized particles. Contrarily, the effect of the refractive index and size distribution on the light scattering seemed to be much stronger in comparison to the morphology of the complex, agglomerate particles. This discovery may simplify the interpretation of photopolarimetric observations of atmospheric aerosols, cosmic dust particles, etc., considerably because the exact specification of the target-particle shape is not required for the analysis (Zubko et al., 2015).

\subsection{Simplifications for Scattering Behavior}

Different theoretical methods are proposed for describing the optical and radiative transfer of the particles and particulate medium. The optical and radiative properties of NPSs can be modeled using the Rayleigh and Lorenz-Mie theories. The Rayleigh scattering is appropriate to tiny, dielectric (non-absorbing), spherical particles. For particles much smaller than the incident wavelength, the Rayleigh theory is suitable. For the Rayleigh scattering, $x \ll 1$ and $|m| x \ll 1$ where $\left(x=\pi d_{p} / \lambda\right)$ is the dimensionless size parameter. The Lorenz-Mie theory provides the general spherical scattering solution (absorbing or non-absorbing) without any particular bound on the particle size, if particle agglomerates are compact and can be defined as effective spheres (Rayleigh, 1870; Mie, 1908; Hulst and van de Hulst, 1957; Mishchenko, 2014; Mishchenko and Dlugach, 2018).

The T-matrix approach is used extensively to obtain the scattering profiles of symmetric particles. It is also known by other names which including the extended boundary condition method (EBCM) and the null-field method (NFM). It was Waterman who first published the T-matrix formulation (Waterman, 1965). In this approach, the matrix is built by matching the boundary conditions to obtain the solutions of the Maxwell equations. To model and formulate the three-dimensional structure and axisymmetric particle, Chebyshev polynomials were used. These are known as Chebyshev particles and the spherical coordinate system is used to give their shape function.

The discrete dipole approximation (DDA) technique is one of the most reliable methods to explain the optical properties of the various nanoparticle agglomerates. It is a numerical method, first proposed by DeVoe (1964), where the calculations were carried out for particles much smaller than the incident wavelength. Then, the formulations were later modified by Purcell and Pennypacker (1973). There are several modifications and new versions of the DDA which were developed by many researchers (Singham and Bohren, 1988; Manickavasagam and Mengüç, 1997; Yurkin et al., 2007). In this approach, the arbitrary shapes of particles and their agglomerates can be modeled as included small dipoles. As expected, when increasing the number of dipoles in a spherical particle of arbitrary size, the solution of the DDA approaches that of the Lorenz-Mie theory. One of the earlier numerical studies was conducted using the discrete dipole approximation approach for closely packed systems containing up to 10 spherical particles in order to explore the inception of soot agglomeration process (Ivezić and Mengüç, 1996; Ivezić et al., 1997; Mishchenko, 2017; Mishchenko and Dlugach, 2018).

There are limitations to the LMT and DDA approaches. The LMT solutions converge very slowly for large values of particle size parameter $(x)$; for example, $x>200$, where $x=\pi d_{p} / \lambda$. The DDA solution is suitable for small values of $x$, and it is computationally expensive and cannot be used for large systems (real NPSs). Simpler methods are preferable to obtain the radiative properties of the particle with the agglomerates and irregular shape. If the particle agglomerates can be correlated to smooth and homogeneous spheres, then the LMT is the best method and could be used for this purpose (Ivezić and Mengüç, 1996; Mishchenko et al., 2017). Although

Volume 27, Issue 3, 2020 
DDA can be used to simulate arbitrary agglomerates with remarkable accuracy, it cannot be easily expanded to large particulate systems. Furthermore, it is reasonable to proceed with a simpler methodology for the calculations for the radiative properties of the particle agglomerates using the LMT, where the properties of the agglomerates can be correlated to smooth, homogeneous spheres (compact particle agglomerates). Figure 3 shows the different shapes of particle agglomerates in the particulate media with the corresponding radiative method; the DDA technique and the Lorenz-Mie theory. $A$ and $\theta$ are the surface at which the electromagnetic energy crosses and the polar angle, respectively (Al-Gebory and Mengüç, 2018).

The LMT and DDA approaches are the general approaches in the most radiative properties calculations for most particulate systems. However, there are important questions such as: "Under what conditions can a particle agglomerate be represented as a homogeneous sphere?", "How can the effects of the multiple shapes, sizes (the particle size distribution) of particle agglomerates with respect to incident radiation be concluded?", and "How can the number of particles in each agglomerate by a number of independent particles be represented?". The answers to all these questions will discover the boundaries between the dependent and independent scattering regimes for the different particle agglomerates.

To date, in the above relationships, multiple and dependent scattering has not been considered. Based on concentration (the nanoparticles number), nanosuspensions can be divided into two categories: diluted and concentrated. The effect of the number of particles should be considered, where increasing particle number leads to a decrease in the clearance of the particles. Therefore, multiple and dependent scattering should be considered and examined. Moreover, in the Rayleigh regime where the particle size is small in comparison to the wavelength, multiple and dependent scattering should be considered also. For the calculation of the radiative properties in terms of the multiple and dependent scattering, related theories such as the quasi-crystalline approximation (QCA) ought to be applied. The QCA, which was introduced by Lax, is a method to explain the multiple and dependent scattering in a dense medium, where the radiative effects between closely packed particles take place (Lax, 1952; Prasher and Phelan, 2005; Han et al., 2018).

Both experimental and analytical analyses are used to calculate the radiative properties of the NPSs. A comparison was done on cases including both fluid base absorbing and non-absorbing

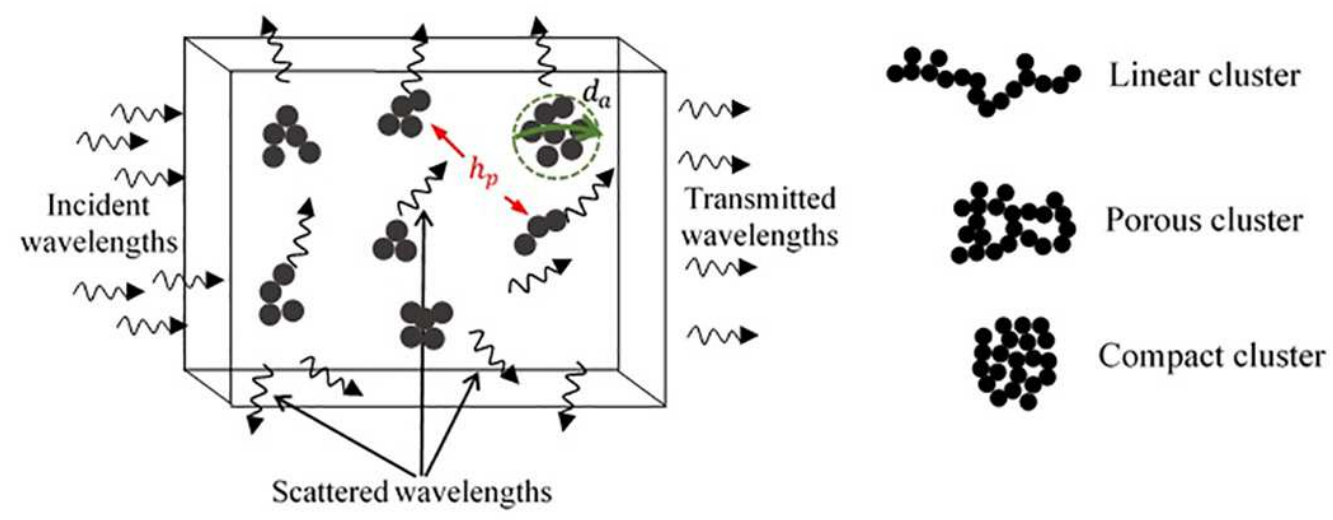

FIG. 3: Incidence of radiative energy on a particulate media includes different particle agglomerates with the corresponding radiative method (adapted from Al-Gebory and Mengüç, 2018) 
matrix, and also for metal and non-metal particles (Wei et al., 2012). Optical and thermal properties (dielectric constant, refractive index, and thermal conductivity, etc.) change with the growth of the nanoparticle size, and some of them affect the individual particles and some affect the clusters. In the field of radiative transfer in NPSs, radiative properties scattering and absorption are for both the base fluid and the nanoparticles. Because of the different sizes and structures of the nanoparticles in the working medium, it is not just a simple summation of their absorption capacity for the nanosuspensions. Scattering is also a loss of intensity of incident light caused by the obstacle particles in the medium, which forces the incident light to be deviated from its straight trajectory (Ortega et al., 2008; Hossain et al., 2015). Spectral transmittance is one of the approaches that allow the determination of the radiative properties. This is used to explain the method by which the incident radiant leaves a surface or medium from a side other than the incident side, which is usually the opposite side. The spectral transmittance refers to the light attenuation and is related to the medium depth and extinction coefficient. Therefore, the calculation of the transmissivity of the NPSs is very important for the study of the effectiveness of solar energy absorption with suspended particles. The spectral transmittance of several NPSs is very different, and these differences depend on different parameters related to the nature of the suspended particles and the medium. Also, the suspended particle size and size distribution play a significant role in the spectral transmittance (Mishchenko, 2014).

$\mathrm{TiO}_{2}$ nanoparticles are used extensively because of their favorable radiative and thermophysical properties, and also because they are very suitable for energy production (solar thermal systems) and water treatment applications (Cheng et al., 2005). To obtain the extinction coefficient and refractive index of water-based $\mathrm{TiO}_{2}$ and $\mathrm{Al}_{2} \mathrm{O}_{3}$ nanofluids, classical theories such as Rayleigh, Maxwell-Garnett, and Lambert-Beer's approaches were used. The extinction coefficient and refractive index of $\mathrm{TiO}_{2}$ nanofluids are found to be higher than $\mathrm{Al}_{2} \mathrm{O}_{3}$ nanofluids in the visible region of light for all the concentrations (Said et al., 2014).

\subsection{Dependent and Independent Scattering}

Light is scattered when its direction is changed by any amount, be it large or small, and this scattering is dependent or independent. Light scattering is always spectral in nature, as it changes with the wavelength of incident light (Manickavasagam and Mengüç, 1997; Modest, 2013; Zhu et al., 2013; Howell et al., 2015).

If the particles are closer to each other, then they scatter the incident light in a dependent fashion, rather than independently, and a correlation between these two regimes can be obtained from experiments or from rigorous theoretical models. More recently, Mishchenko et al. proposed a rigorous approach for radiative transfer in packed systems following the fundamental of EM-wave scattering (Mishchenko et al., 1999; Taylor et al., 2011; Mishchenko and Yurkin, 2017). However, for most practical systems involving thousands of particles in agglomerates or in dispersions, a simpler approach is needed. Such an approach is called a dependent scattering approach. In a particulate media, the particle number, size, and size should be examined to explore the dependent and independent scattering. The number of particles and size affect the distance between the particles and its relationship with the incident wavelength has a significant effect on the dependent and independent scattering (Prasher and Phelan, 2005; Otanicar et al., 2009; Swamy et al., 2009). Dependent scattering is introduced into the radiative properties by two mechanisms. The first is the near field (the near-field interparticle) effect due to the multiple scattering at the internal fields of the particles, which changes both the extinction and scattering properties of the system. The second effect comes from the far field which is manifested by a

Volume 27, Issue 3, 2020 
change in the scattering properties only. The dependent scattering has significant impacts on the radiative transfer in particulate suspensions (Agrawal and Mengüç, 1991; Aslan et al., 2006a). In compact NPSs (particles are closely and neatly packed together; dense), the dependent scattering should be considered and examined, while for loose NPSs (particles are separated with considerable clearance; non-dense), the independent scattering can be suggested.

The effects of dependent scattering become more important as the particle concentration increases, at which point the clearance between the particles decreases, and they become close to each other. For independent scattering, the conditions of $\phi<0.6 \%$ or $h_{\text {sur, }} / \lambda>0.5$ are required, where $\lambda$ is the wavelength (Modest, 2013; Howell et al., 2015). The boundaries of the dependent and independent scattering regimes were first demarcated by Drolen and Tien, as shown in Fig. 4 (Drolen and Tien, 1987). A wide range of particulate media at different particle volume fractions and size parameters was covered by these boundaries. Then, different investigations were carried out on the boundaries of the dependent and independent scattering regimes (DISRs) for different media in various conditions, where the effects of the different parameters were included, as shown in Figs. 5 and 6 (Aslan et al., 2006a; Otanicar et al., 2009). The condition $\left(h_{\text {sur }, p} / \lambda\right)$ separates between the boundaries of the DISRs, and radiative scattering occurs in the independent scattering regime when the $h_{\text {sur }, p} / \lambda$ value is larger than 0.5 . However, when the value of $h_{\text {sur }, p} / \lambda$ is below 0.5, the NPSs fall into the Rayleigh regime. This is when dependent scattering needs be considered. In other words, for an independent scattering case, the distance between the particle to particle surfaces should be large enough in comparison with the incident wavelength to overcome the effect of the scattering from the other particles.

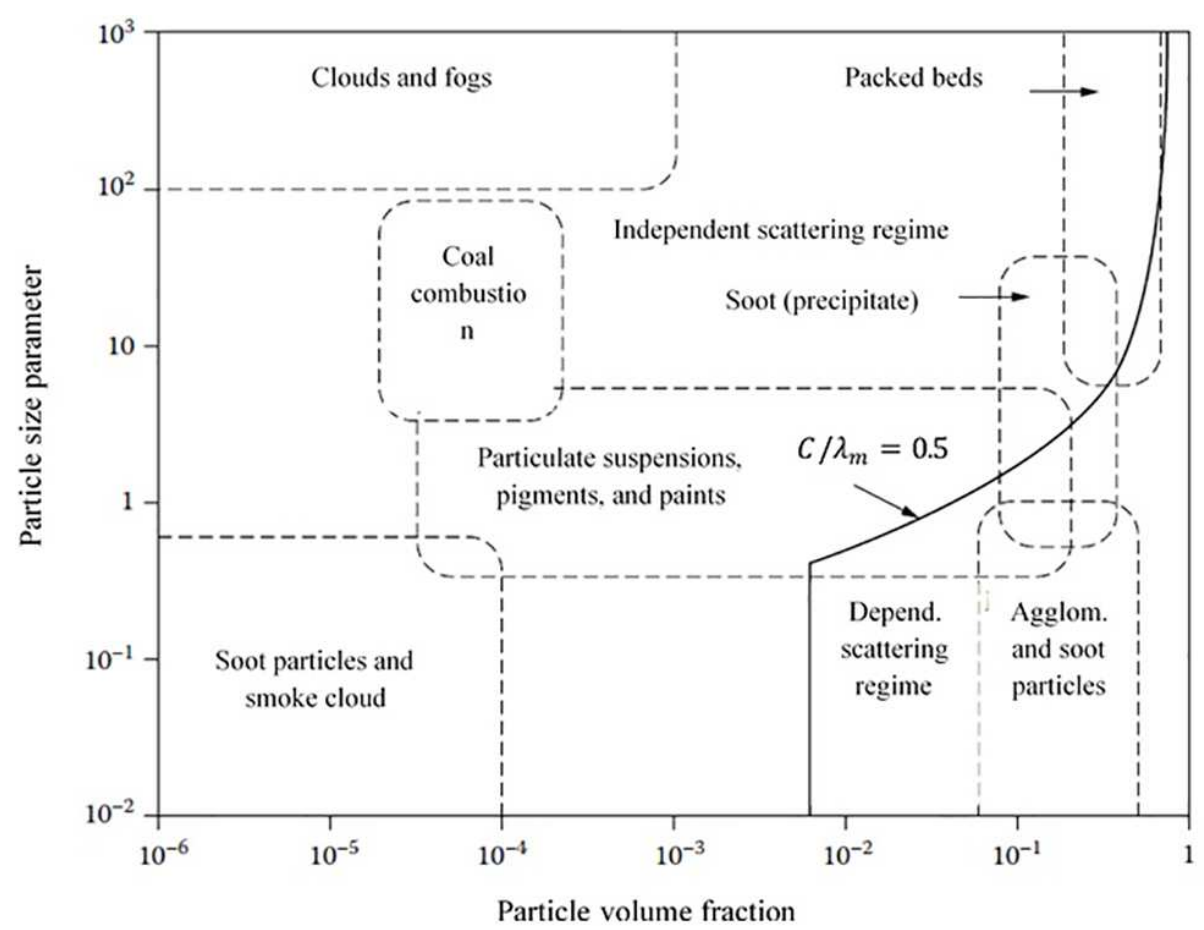

FIG. 4: Map of independent and dependent scattering regimes as a function of particle size parameter and volume fraction (see Drolen and Tien, 1987 for more details) 


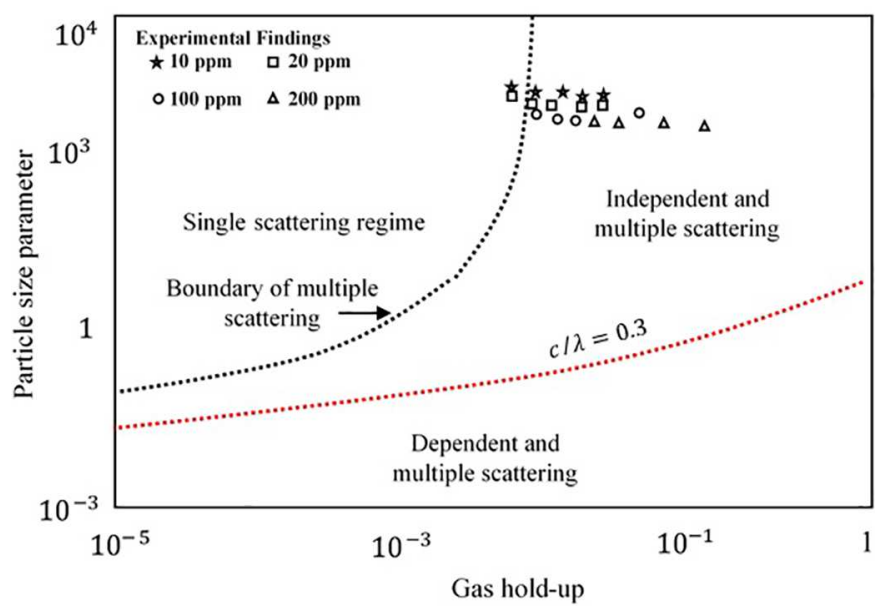

FIG. 5: Single and multiple (independent and dependent) scattering regimes for bubbles in the liquid as a function of size parameter and gas hold-up, experimental results are shown for the present system (adapted from Aslan et al., 2006a)

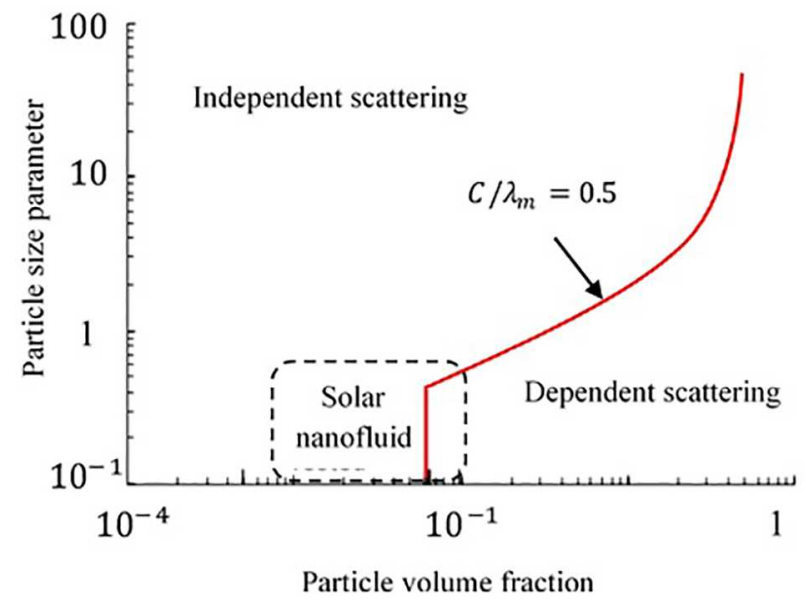

FIG. 6: Scattering regime map for solar nanofluids (see Otanicar et al., 2009 for more details)

The stability of the NPSs is a very important parameter. As the particles agglomerate, or as they settle down, their interaction with radiation can be more complicated and cannot be predicted. The effects of different parameters $\left(\mathrm{pH}, d_{p}\right.$, particle shape, $h_{s u r, p}$, and $\left.\emptyset\right)$ have been investigated on how the particles scatter radiation, and to determine if the dependent or independent scattering regimes can be used to determine the radiative properties of the NPSs. Indeed, for a particular particulate media, different boundaries can be specified by adjusting the $\mathrm{pH}$ at different values, where various particle agglomeration behaviors are obtained. Including the effect of the $\mathrm{pH}$ and the isoelectric point of particles in the boundaries of the DISRs serve as important indications in the scattering (dependent and independent) and can be utilized in a wide range of practical applications (Al-Gebory and Mengüç, 2018). Most of the studies on the radiative properties of the NPSs are completed without taking the effects of the dependent/independent

Volume 27, Issue 3, 2020 
scattering into consideration (Dietz, 2004; Muhammad et al., 2016). Because of this, a detailed discussion of the dependent and independent scattering concept is required.

The boundaries of the DISRs were first demarcated by Drolen and Tien. They presented the fundamentals of the scattering and absorption of thermal radiation in particulate systems. The common assumption is that each particle in the system scatters and absorbs radiation, which is not affected by the presence of others, is the "independent" assumption. The departure of the net incidence on each particle because of the radiation scattered from the others, and the interference in the far field due to the phase addition of the scattered electric fields from the particles. The first causes an increase in energy extinguished and scattered while the second leads to a decrease in the total scattered energy (Drolen and Tien, 1987). Several light-scattering techniques have been used over the years to measure the optical and radiative properties of nanoparticles and agglomerates. Dependent and independent scattering regimes have been investigated by many researchers, where the effects of different parameters were explored (Aslan et al., 2006a; Otanicar et al., 2009), as shown in Figs. 5 and 6.

Al-Gebory and Mengüç studied the effect of the $\mathrm{pH}$ value on the dependent and independent scattering of $\mathrm{TiO}_{2}$ NPSs as shown in Fig. 7. In this figure, adjusting the $\mathrm{pH}$ value far from the $\mathrm{pH}_{i s o}$ of a particle may produce ultrafine particles and/or small size agglomerates; the dependent scattering may occur at this value of $\mathrm{pH}$ (Al-Gebory and Mengüç, 2018). An earlier numerical study was carried out using the discrete dipole approximation method for closely packed systems containing up to 12 spherical particles so as to investigate the inception of agglomeration. The effects of dependent scattering in a medium consisting of two spheres at an arbitrary distance between them and the index of refraction typical for that of soot particles were investigated (Ivezić and Mengüç, 1996; Wang and Zhao, 2018).

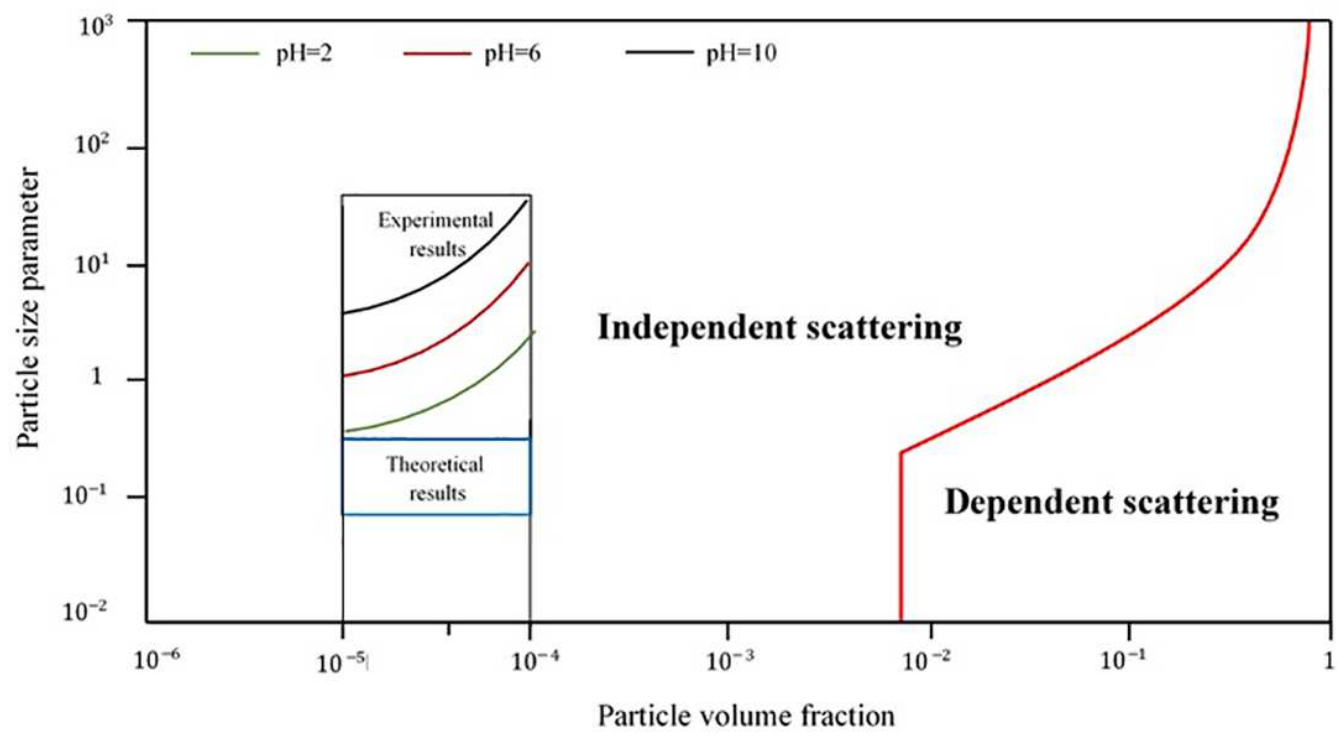

FIG. 7: The boundaries of independent and dependent scattering regimes for $\mathrm{TiO}_{2} \mathrm{NPSs}$. The experimental results based on the DLS technique at different $\emptyset$ and $\mathrm{pH}$ values (regarding to particle agglomeration) and the theoretical results (regardless of article agglomeration) are included (adapted from Al-Gebory and Mengüç, 2018). 


\subsection{Radiative Properties of Nanoparticle Suspensions}

The interaction of electromagnetic radiation with the nanoparticles and their agglomerates in the NPSs can be represented by the optical and radiative properties. These properties depend on (i) the shape of the nanoparticle, (ii) the material of the nanoparticle (i.e., the complex index of refraction, $m=\bar{n}+i \bar{k}$ ), (iii) its relative size, and (iv) the clearance between the nanoparticles. For the interaction of the radiation with a sphere, the efficiency factors $\left(Q_{i}=C_{i} / \pi r_{p}^{2}\right)$ are formulated by cross sections $C_{i}$ normalized to the projected surface area $\left(\pi r_{p}^{2}\right)$, where $i$ refers to scattering $(i=s c a t)$, absorption $(i=a b s)$, and extinction $(i=e x t)$. Then, the radiative efficiencies are defined as $Q_{e x t}=C_{e x t} /\left(\pi r_{p}^{2}\right), Q_{a b s}=C_{a b s} /\left(\pi r_{p}^{2}\right)$ and $Q_{s c a t}=C_{s c a t} /\left(\pi r_{p}^{2}\right)$. In the above relationships, the particle radius and diameter $\left(r_{p}\right.$ and $\left.d_{p}\right)$ and particle number can be replaced by the particle agglomerate radius and diameter $\left(R_{a g g}\right.$ and $\left.d_{a g g}\right)$ if the spherical shape of particle agglomerates can be obtained (Modest, 2013; Howell et al., 2015). The nanoparticles in the suspension show a different particle size under the effect of agglomeration. One can clarify the condition of the scattering phenomena (dependent or independent), so the radiative properties can be modeled accurately. In the case of independent scattering, the radiative properties of the nanoparticles or their agglomerates can be obtained by using the linear summation role (the radiative properties of a single particle multiplied by the number of particles). The scattering and absorption coefficients of the cloud nanoparticles can be calculated as $\sigma_{\text {scat }}=N Q_{\text {scat }}$ and $\kappa_{a b s}=N Q_{a b s}$, where $N$ represents the number of particles per unit volume. This role is applicable for independent scattering; it is also applicable and valid for many applications considered in the field of radiative transfer (Howell et al., 2015).

The radiative properties of NPs and NPSs have been investigated extensively in recent years, and the effects of different nanoparticle types, in addition to their physical and chemical characteristics effects on their radiative properties and radiative transfer, have been observed (Viskanta and Mengüç, 1989; Wei et al., 2012; Said et al., 2013). Figure 8 shows the scattering coefficients of different nanoparticle and nanotube suspensions under different conditions which have been collected from different studies. Figure 8(a) shows the scattering coefficient of $\mathrm{Al}_{2} \mathrm{O}_{3}$ nanoparticle suspensions. Said et al. shows the scattering coefficient of water $/ \mathrm{Al}_{2} \mathrm{O}_{3}$ NPSs using the Rayleigh approach; the surfactants (sodium dodecyl sulfate and polyvinylpyrrolidone) were used for stability enhancement. The size of the $\mathrm{Al}_{2} \mathrm{O}_{3} \mathrm{NP}$ was $d_{p}=13 \mathrm{~nm}$ and the particle volume fraction was $0.1 \%$ and $0.3 \% v / v$ (Said et al., 2014). Sajid et al. shows the scattering coefficient of water $/ \mathrm{Al}_{2} \mathrm{O}_{3}$ NPSs, and the results were taken using the Rayleigh approach because its applicability is in low concentration colloidal suspensions. The particle size was $d_{p}=1 \mathrm{~nm}$ and the particle volume fraction was $0.08 \%$ and $0.03 \% v / v$ (Sajid et al., 2014). Al-Gebory et al. shows the scattering coefficient of water $/ \mathrm{Al}_{2} \mathrm{O}_{3}$ NPSs (a $40 \mathrm{~nm}$ diameter size) using the Lorenz-Mie theory, and the results were taken at different $\mathrm{pH}$ values without using any type of surfactant. Different concentrations of the NP were used to obtain the scattering coefficient considering the compact (spherical) particle agglomerate based on the $\mathrm{pH}_{\text {iso }}$ of $\mathrm{Al}_{2} \mathrm{O}_{3} \mathrm{NP}$ (Al-Gebory et al., 2018). Figure 8 (b) represents the scattering coefficient of $\mathrm{TiO}_{2}$ nanoparticle suspensions. Said et al. shows the scattering coefficient of water/ $/ \mathrm{TiO}_{2}$ NPSs using the Rayleigh approach. The size of the $\mathrm{TiO}_{2}$ NP was $d_{p}=21 \mathrm{~nm}$ and the particle volume fraction was $0.1 \%$ and $0.3 \% v / v$ (Said et al., 2014). Tan et al. shows the scattering coefficient of water/ $/ \mathrm{TiO}_{2}$ NPSs. The Monte Carlo method combined with the Mie scattering phase function was developed to calculate the optical properties and radiative transfer in the NPSs. The $\mathrm{TiO}_{2}$ NP size was $\left(d_{p}=20,60\right.$, and $\left.600 \mathrm{~nm}\right)$ and the particle volume fraction was $0.005 \% v / v$ (Tan et al., 2017). Al-Gebory et al. shows the scattering coefficient of water/ $/ \mathrm{TiO}_{2}$ NPSs, the particle size was $40 \mathrm{~nm}$, and the effect of the $\mathrm{pH}$ value

Volume 27, Issue 3, 2020 


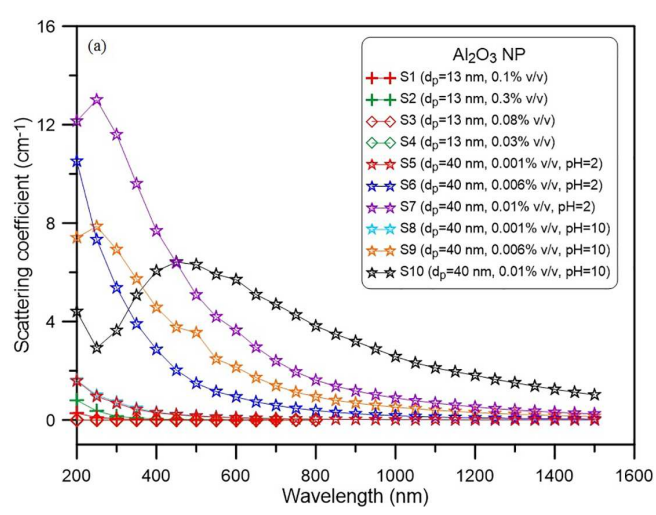

(a)

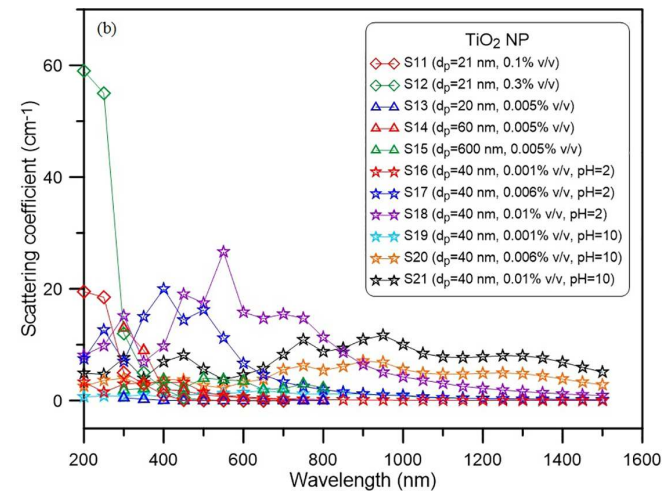

(b)

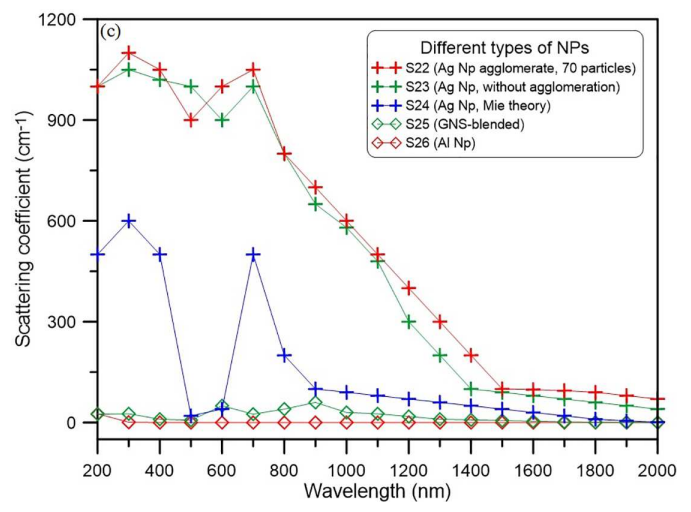

(c)

FIG. 8: Scattering coefficient profiles for different types of NPSs. (a) $\mathrm{Al}_{2} \mathrm{O}_{3}$ NP-based NpSs, $\mathrm{S} 1$ and $\mathrm{S} 2$ (Said et al., 2014), S3 and S4 (Sajid et al., 2014), S5-S10 (Al-Gebory et al., 2018). (b) $\mathrm{TiO}_{2} \mathrm{NP}$-based NpSs, S11 and S12 (Said et al., 2014), S13-S15 (Tan et al., 2017), S16-S21 (Al-Gebory et al., 2018). (c) Different types of NPs-based NpSs, S22-S24 (Du and Tang, 2015), S25-S26 (Lee et al., 2012).

on the scattering coefficient was shown using the Lorenz-Mie theory (Al-Gebory et al., 2018). Figure 8(c) shows the scattering coefficient of different types of nanoparticle suspensions under different conditions. Du and Tang proposed a theoretical approach for calculating the radiative properties of water/Ag NPSs with particle agglomeration based on diffusion-limited cluster agglomeration (DLCA) simulation and the generalized multiparticle Mie solution (GMM) method. The investigation was carried out on the NPSs with the particle volume fraction $(\emptyset=1 \% v / v)$ and the $\mathrm{Ag}$ particle diameter was $30 \mathrm{~nm}$. The calculations were conducted for three cases; the particle agglomerate with 70 particles, nanoparticle without considering the agglomeration effect, and the single particle using the Lorenz-Mie theory (Du and Tang, 2015). Lee et al. shows the scattering coefficient of the gold-nanoshell (GNS) nanoparticle with different $\mathrm{SiO}_{2}$ sizes and the Au coating thickness are mixed in water to make a GNS blended with particle volume fraction of $0.07 \% v / v$. The scattering coefficient of the Al/water NPSs was with the particle volume fraction of $0.07 \% \mathrm{v} / \mathrm{v}$. A Monte Carlo algorithm combined with the Mie scattering theory is developed to determine the radiative properties (Lee et al., 2012). 
The absorption coefficient of different nanoparticles and nanotube suspensions is shown in Fig. 9. Figure 9(a) shows the absorption coefficient of $\mathrm{Al}_{2} \mathrm{O}_{3}$ nanoparticle suspensions. Said et al. shows the scattering coefficient of water $/ \mathrm{Al}_{2} \mathrm{O}_{3}$ NPSs using the Rayleigh approach, and the effect of the particle stability on the absorption coefficient is shown. The size of the $\mathrm{Al}_{2} \mathrm{O}_{3} \mathrm{NP}$ was $d_{p}=13 \mathrm{~nm}$ and the particle volume fraction was $0.1 \%$ and $0.3 \% v / v$ (Said et al., 2014). Sajid et al. shows the absorption coefficient of water $/ \mathrm{Al}_{2} \mathrm{O}_{3} \mathrm{NPSs}$, and the results were taken at $25^{\circ} \mathrm{C}$ using the Rayleigh approach. The particle size was $d_{p}=13 \mathrm{~nm}$ and the particle volume fraction was $0.03 \%, 0.05 \%$, and $0.08 \% v / v$ (Sajid et al., 2015). Figure 9(b) represents the absorption coefficient of $\mathrm{TiO}_{2}$ nanoparticle suspensions. Jianya et al. shows the absorption coefficient of water/TiO ${ }_{2}$ NPSs. The Monte Carlo method combined with the Mie scattering phase function was developed to calculate the optical properties and radiative transfer in the $\mathrm{NPSs}^{\mathrm{TiO}} \mathrm{T}_{2} \mathrm{NP}$ size was $\left(d_{p}=20,60\right.$, and $\left.600 \mathrm{~nm}\right)$ and the particle volume fraction was $0.005 \% v / v$ (Tan et al., 2017). Said et al. shows the absorption coefficient of water/ $\mathrm{TiO}_{2} \mathrm{NPSs}$ using the Rayleigh approach. Different size of particles were used $\left(d_{p}=1,5,10\right.$, and $\left.20 \mathrm{~nm}\right)$ and the particle volume fraction was $0.05 \% v / v$ (Said et al., 2015). Figure 9(c) shows the scattering coefficient of

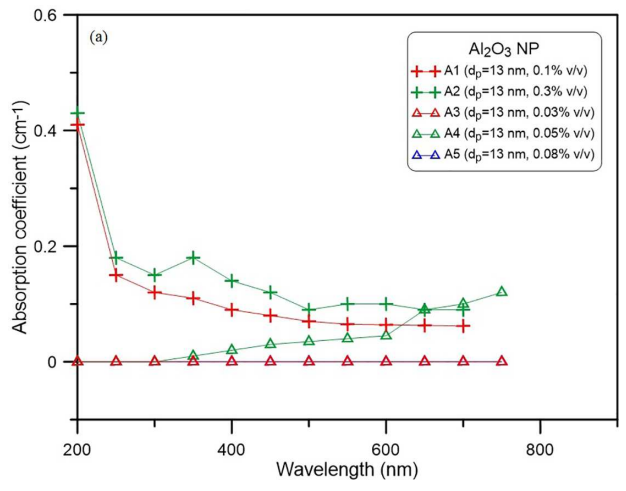

(a)

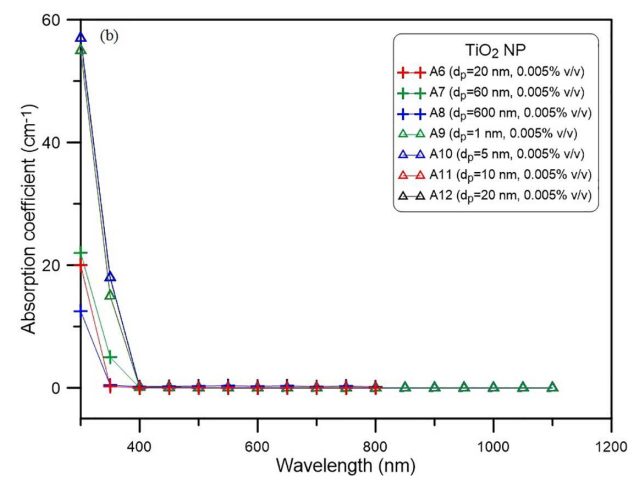

(b)

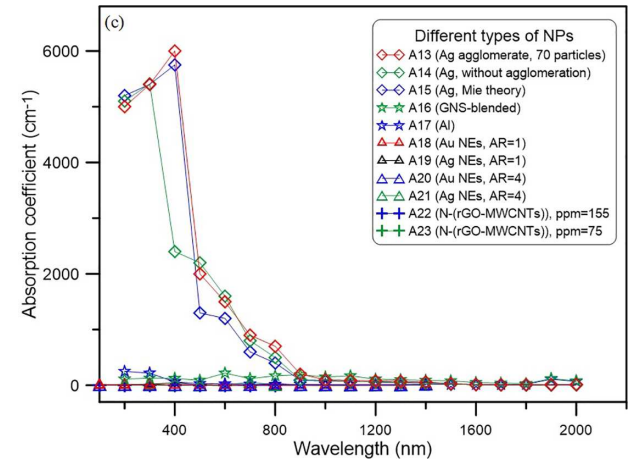

(c)

FIG. 9: Absorption coefficient profiles for different types of NPSs. (a) $\mathrm{Al}_{2} \mathrm{O}_{3}$ NP-based NpSs, $\mathrm{A} 1$ and $\mathrm{A} 2$ (Said et al., 2014), A3-A5 (Sajid et al., 2015). (b) $\mathrm{TiO}_{2}$ NP-based NpSs, A6-A8 (Tan et al., 2017), A9A12 (Said et al., 2015). (c) Different types of NPs-based NpSs, A13-A15 (Du and Tang, 2015), A16 and A17 (Lee et al., 2012), A18-A21 (Rativa and Gómez-Malagón, 2015), A22 and A23 (Shende and Sundara, 2015).

Volume 27, Issue 3, 2020 
different types of nanoparticle suspensions under different conditions. Du and Tang proposed a theoretical approach for calculating the absorption coefficient of water/Ag NPSs under different conditions with and without particle agglomeration using the Lorenz-Mie theory (Du and Tang, 2015). Lee et al. shows the absorption coefficient of the gold-nanoshell (GNS) nanoparticle with different $\mathrm{SiO}_{2}$ sizes and the Au coating thickness are mixed in water to make a GNS blended with particle volume fraction of $0.07 \% v / v$ and the absorption coefficient of Al/water NPSs with particle volume fraction of $0.07 \% \mathrm{v} / \mathrm{v}$. A Monte Carlo algorithm combined with the Mie scattering theory is developed to determine the radiative properties (Lee et al., 2012). Rativa and Gómez-Malagón studied the linear optical absorption in the visible and near-IR spectral region of the aqueous nanofluid containing gold and silver nanoellipsoids (NEs) using the MaxwellGarnett model. The results show an enhancement of the absorption coefficient in almost 54\% and $86 \%$ for gold and silver NEs. Au and Ag NEs were with the aspect ratio AR $=1$ and 4 (Rativa and Gómez-Malagón, 2015). Shende and Sundara studied the absorption coefficient of the nitrogen-doped hybrid structure of reduced graphene oxide [N-(rGO-MWCNTs)]. The absorption coefficient was carried out using a UV-visible-NIR spectrophotometer. The concentrations of the N-(rGO-MWCNTs) were ppm = 155 and 75 (Shende and Sundara, 2015).

Figure 10 shows the extinction coefficient of the different types of nanoparticle suspensions under different conditions. Figure 10(a) shows the extinction coefficient of the $\mathrm{Al}_{2} \mathrm{O}_{3}$ nanoparticle suspensions. Said et al. shows the extinction coefficient of water $/ \mathrm{Al}_{2} \mathrm{O}_{3}$ NPSs using the Rayleigh approach and the experimental method (UV-vis-NIR spectrophotometer), and the size of the $\mathrm{Al}_{2} \mathrm{O}_{3} \mathrm{NP}$ was $d_{p}=13 \mathrm{~nm}$ and the particle volume fraction was $0.1 \%$ and $0.3 \% v / v$ (Said et al., 2014). Menbari and Alemrajabi studied the extinction coefficient of $\mathrm{Al}_{2} \mathrm{O}_{3} \mathrm{NP}(0.04 \% v / v)$ using the UV-vis-NIR spectrophotometer (Menbari and Alemrajabi, 2016). Al-Gebory et al. shows the extinction coefficient of water/ $\mathrm{Al}_{2} \mathrm{O}_{3}$ NPSs using the Lorenz-Mie theory, and the results were taken at different $\mathrm{pH}$ values without using any type of surfactant. Different concentrations of NP were used to obtain the scattering coefficient considering the compact (spherical) particle agglomerate based on the $\mathrm{pH}_{\text {iso }}$ of $\mathrm{Al}_{2} \mathrm{O}_{3} \mathrm{NP}$ (Al-Gebory et al., 2018). Figure 10(b) represents the extinction coefficient of $\mathrm{TiO}_{2}$ nanoparticle suspensions. Said et al. shows the extinction coefficient of water/TiO ${ }_{2}$ NPSs using a UV-vis-NIR spectrophotometer, and the surfactants (sodium dodecyl sulfate and polyvinylpyrrolidone) were used for stability enhancement. The size of the $\mathrm{TiO}_{2} \mathrm{NP}$ was $d_{p}=21 \mathrm{~nm}$ and the particle volume fraction was $0.1 \%$ and $0.3 \%$ $v / v$ (Said et al., 2014). Al-Gebory et al. shows the extinction coefficient of water/TiO ${ }_{2} \mathrm{NPSs}$ under different $\mathrm{pH}$ values using the Lorenz-Mie theory, and the results were taken at different $\mathrm{pH}$ values without using any type of surfactant (Al-Gebory et al., 2018). Figure 10(c) shows the scattering coefficient of different types of nanoparticle suspensions under different conditions. $\mathrm{He}$ et al. shows the extinction coefficient of water/Cu NPSs using the Rayleigh approach and the experimental method (UV-vis-NIR spectrophotometer). The size of the Cu NP was $d_{p}=50 \mathrm{~nm}$ and with different particle mass fractions was $0.1 \%, 0.04 \%, 0.02 \%$, and $0.01 \%$ wt. (He et al., 2013). Shende and Sundara studied the extinction coefficient of the nitrogen-doped hybrid structure of reduced graphene oxide [N-(rGO-MWCNTs)]. The absorption coefficient was carried out using a UV-visible-NIR spectrophotometer. The concentrations of the N-(rGO-MWCNTs) were ppm $=155$ and 75 (Shende and Sundara, 2015). Karami et al. studied the extinction coefficient of aqueous suspensions based on alkaline functionalized CNT (f-CNT) using a UV-visNIR spectrophotometer. The concentrations of the $\mathrm{f}-\mathrm{CNT}$ were $5 \%$ and $10 \% \mathrm{ppm}$ (Karami et al., 2014). Menbari and Alemrajabi studied the extinction coefficient of $\mathrm{CuO} /$ water $(0.001 \% \mathrm{v} / \mathrm{v})$ and $\mathrm{Al}_{2} \mathrm{O}_{3}+\mathrm{CuO} /$ water $\left(0.004 \% v / v \mathrm{Al}_{2} \mathrm{O}_{3}+0.001 \% v / v \mathrm{CuO}\right)$ using a UV-vis spectrophotometer (Menbari and Alemrajabi, 2016). 


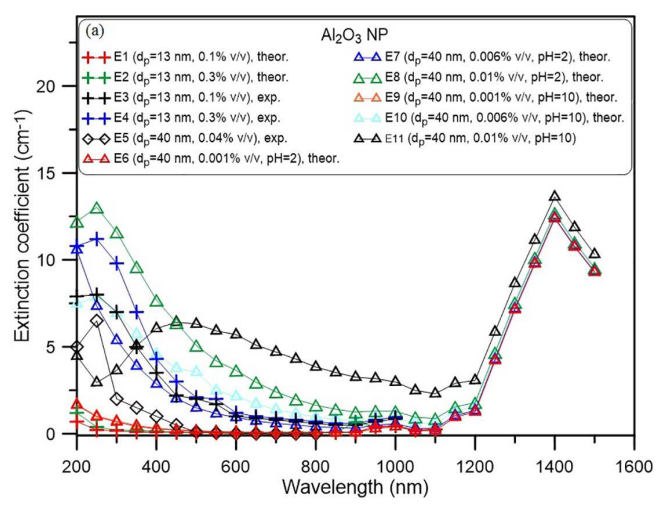

(a)

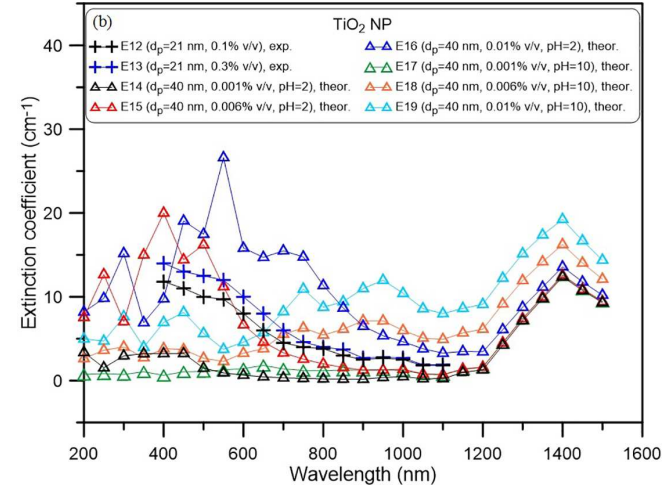

(b)

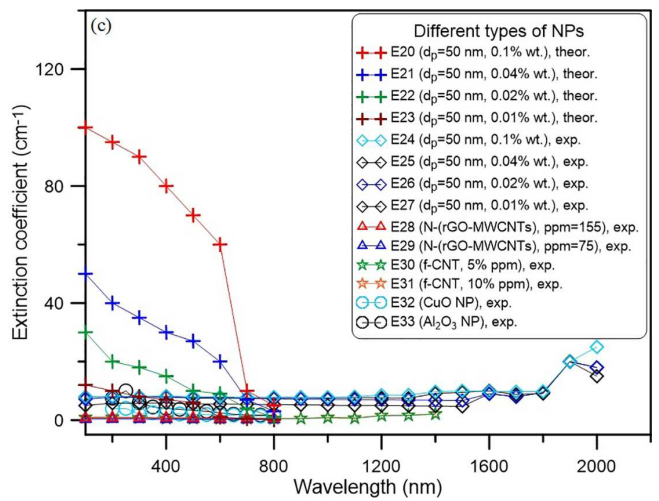

(c)

FIG. 10: Extinction coefficient profiles for different types of NPSs. (a) $\mathrm{Al}_{2} \mathrm{O}_{3}$ NP-based NpSs, E1-E4 (Said et al., 2014), E5 (Menbari and Alemrajabi, 2016), E6-E11 (Al-Gebory et al., 2018). (b) $\mathrm{TiO}_{2} \mathrm{NP}_{\text {-based }}$ NpSs, E12 and E13 (Said et al., 2014), E14-E19 (Al-Gebory et al., 2018). (c) Different types of NPs-based NpSs, E20-E27 (He et al., 2013), E28 and E29 (Shende and Sundara, 2015), E30 and E31 (Karami et al., 2014), E32 and E33 (Menbari and Alemrajabi, 2016).

The results for the radiative properties of nanoparticle suspensions show that the stability of nanoparticles have significant effects. The different parameters (nanoparticle type and size, optical properties of the nanoparticle and the medium, preparation methods, the stability and agglomeration of the particles) can produce different radiative properties. Particle agglomeration can improve light attenuation inside nanosuspensions; this leads to improve radiative absorptivity and as a result transmissivity of nanosuspensions is reduced compared with no particle agglomeration. Then the existence of particle agglomeration can be able to enhance the radiative properties of thermal radiation systems.

\subsection{Radiation Transmittance in Nanoparticle Suspensions}

Incident radiation on suspensions is redirected by scattering, where deviated or redirected radiation passes through multiple scattering in particulate media and can be absorbed by particles and/or the medium. These concepts represent radiation augmentation and attenuation.

Volume 27, Issue 3, 2020 
The attenuation of incident radiation (extinction coefficient) in NPSs can be calculated from the Beer-Lambert law (Swinehart, 1962). In this law, the attenuation of radiation is given by $T=I / I_{0}=e^{-l \sigma_{\text {total }}}$, where $T$ is the spectral transmittance, $I$ and $I_{0}$ represent the transmitted and incident radiation intensity, $l$ is the length along the incident direction, and $\sigma_{\text {total }}$ is the total extinction coefficient of NPSs. A lower percentage of transmittance indicated that the nanosuspension is a good solar absorber and has a better solar absorption ability. A suspended particle in a media produces particle agglomerates with different sizes and structures in addition to the particle size distribution. Therefore, there is some difficulty in explaining all these aspects theoretically. The added effect is that the agglomeration of differing particle kinds is in the same medium when a hybrid particulate system is recommended, in which a particle agglomerate shows the properties (the chemical, physical, and radiative, etc.) of the different materials that constructed such a particle agglomerate, where each particle type has its own properties. This becomes an additional effect besides the others from different sizes and structures. For that, experimental investigations are required in order to realize these aspects and their effect on radiative properties and transfer. The majority of experimental research regarding radiative properties are based on spectrophotometers or self-designed apparatus in accordance with the Lambert-Beer law. Spectrophotometers are used to obtain the optical and radiative properties of the NPSs including absorption, scattering, and extinction properties (Lee and Jang, 2013; Duan and Xuan, 2014; Gorji et al., 2015).

Even though spectrophotometers have been applied; some extra treatments are still needed due to the following two reasons. Firstly, because NPSs samples need to be dispersed into a glass or plastic container, and the transmission and reflection of the container walls will be coupled with the optical properties of the NPSs. This is shown in Fig. 11 (Taylor et al., 2011). In this figure, $\sigma$ and $\mathrm{k}$ represent the scattering and absorption coefficients. Because a cuvette includes the liquid sample in the system, the resulting measurement is in fact a "three-slab system." This adds complexity since there can be multiple reflections at each interface which need to be taken into account in the measurements. As is seen in Fig. 11, some of the signals going through the three-slab system are lost in the reflections at the interfaces. Secondly, the NPSs are two-phase mixtures, i.e., inhomogeneous medium, and light transporting in an inhomogeneous (heterogeneous) medium may accompany the scattering, which leads to all the directions' diffusion other than the incident direction (Song et al., 2016). Thus, the acquired absorption coefficient always includes the contribution of some scattering light. Despite the spectrophotometer used, some

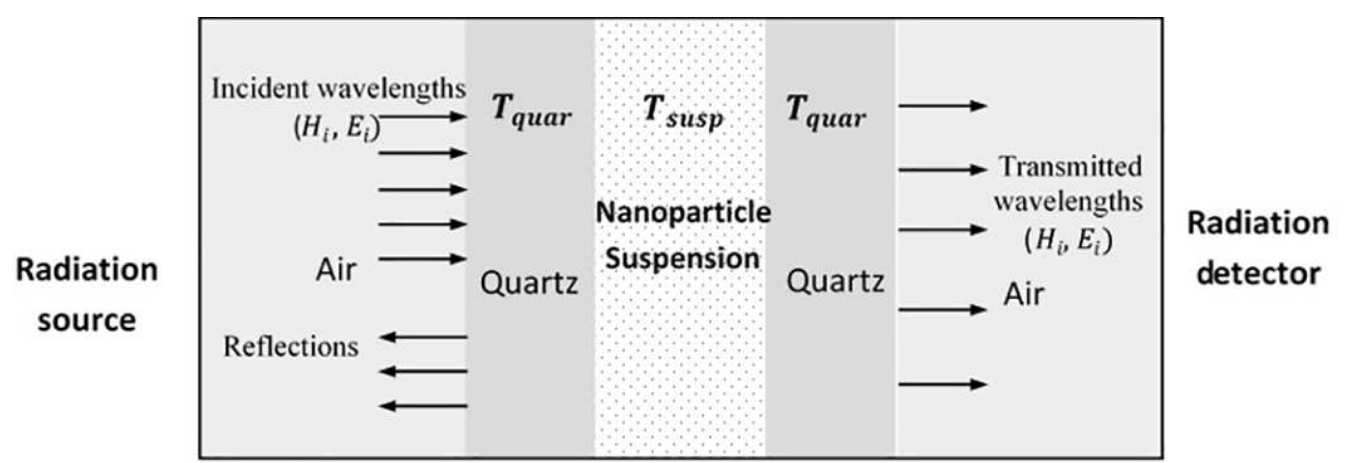

FIG. 11: Diagram of three-slab system representation for a spectrometry measurement of a NPS-filled quartz cuvette (adapted from Taylor et al., 2011) 
other calculations are required in order to attain the extinction coefficients for the NPSs. Even though the two issues always exist in the measurement of the optical coefficients of NPSs, under particular conditions, either one or both of them may be negligible, which then means the experimental design can be made simpler. Spectral transmittance is the term used to describe the process by which incident radiant leaves a surface or medium from a side other than the incident side, usually the opposite side. The spectral transmittance is related to the medium depth and medium extinction coefficient. The scattering contribution increases as the particles agglomerate to a greater extent (particle agglomerate size); the structure of the agglomerates also plays a significant role in the light attenuation, and as a result, it affects the spectral transmittance of the incident radiation (Kameya and Hanamura, 2011; Said et al., 2014).

The spectral transmittance of the different types of nanoparticle suspensions is shown in Fig. 12. Figure 12(a) shows the spectral transmittance of carbon nanotubes and carbon nanohorn suspensions. Sani et al. show the spectral transmittance of single-wall carbon nanohorns in water with different concentrations $(0.001$ and $0.01 \mathrm{~g} / \mathrm{l})$. The spectra transmittance was measured at room temperature using a double-beam spectrophotometer (Perkin-Elmer Lambda 900) (Sani et al., 2010). Mercatelli et al. shows the spectral transmittance of single-wall carbon nanohorns in aqueous (water) and glycol-based nanofluids with concentrations (0.005 g/l). Karami et al. shows the spectral transmittance of $\mathrm{f}-\mathrm{CNT} /$ water with different concentrations ( 5 and $50 \% \mathrm{ppm}$ ). The UV-vis-NIR spectrophotometer (Perkin-Elmer Lambda 900) was used for this purpose (Mercatelli et al., 2011). Luo et al. shows the spectral transmittance of short carbons (OD = 10-20 $\mathrm{nm}$, length $=0.5-2 \mu \mathrm{m})$. The medium was Texatherm oil to produce stable NPSs. The UV-vis spectrophotometer (Shimadzu UV3150) was used for the radiation transmittance measurements (Luo et al., 2014). He et al. shows the spectral transmittance of hybrid nanomaterial, single-wall carbon nanotube-copper sulfide nanoparticles (SWCNT-CuS NPs) in a poly (styrene sulfonate) solution. The concentration was $\left(0.01 \mathrm{mg} \mathrm{ml}^{-1} \mathrm{SWCNT}\right)$. A spectrophotometer is used for the spectral transmittance measurements (He et al., 2014). Figure 12(b) shows the spectral transmittance of metal nanoparticle suspensions.

$\mathrm{Du}$ and Tang used a theoretical approach for calculating the radiative properties of water/Ag NPSs with particle agglomeration based on the diffusion-limited cluster agglomeration (DLCA) simulation and generalized multiparticle Mie solution (GMM) method. The investigation was carried out on the NPSs with the particle volume fraction $(\emptyset=1 \% v / v)$ and the $\mathrm{Ag}$ particle diameter was $30 \mathrm{~nm}$. The calculations were carried out for three cases; particle agglomerate with 70 particles, nanoparticle without considering the agglomeration effect, and single particle using the Lorenz-Mie theory (Du and Tang, 2015). Luo et al. shows the spectral transmittance of $\mathrm{Ag}$ and $\mathrm{Cu}$ NPs $\left(d_{p}=50 \mathrm{~nm}\right)$ in Texatherm oil. The Ag nanoparticle concentration was $(0.1 \%$ $v / v)$ and $\mathrm{Cu}$ nanoparticle concentration was $(1 \% v / v)$ (Luo et al., 2014). Zhu et al. studied the radiation transmittance of AIN/water (40 nm size) with nanoparticle concentration $(0.02 \%$ wt.), $\mathrm{ZrC} /$ water (40 $\mathrm{nm}$ size) with the nanoparticle concentration $(0.02 \% \mathrm{wt}$.), and TiN/water (20 $\mathrm{nm}$ size) with the nanoparticle concentration $(0.02 \% \mathrm{wt}$.). A spectrophotometer was used for the spectral transmittance measurements (Zhu et al., 2013). He et al. shows the spectral transmittance of copper sulfide nanoparticles (CuS NPs) in poly (styrene sulfonate) solution. The concentration was $\left(0.01 \mathrm{mg} \mathrm{ml}^{-1}\right)$. A spectrophotometer is used for the spectral transmittance measurements (He et al., 2014). Zhang et al. studied the spectral transmittance of $\mathrm{Ni}(40 \mathrm{~nm})$ and $\mathrm{Cu}(40 \mathrm{~nm})$ in water, and the nanoparticle concentration was $10 \% v / v$. UV-vis-NIR spectrophotometer was used for this purpose (Zhang et al., 2014). Figure 12(c) shows the spectral transmittance of metal oxide nanoparticle suspensions. Zhu et al. shows the spectral transmittance of $\mathrm{ZnO} /$ water NPSs; the particle size was $10 \mathrm{~nm}$ with a concentration of $0.02 \%$ wt. and

Volume 27, Issue 3, 2020 


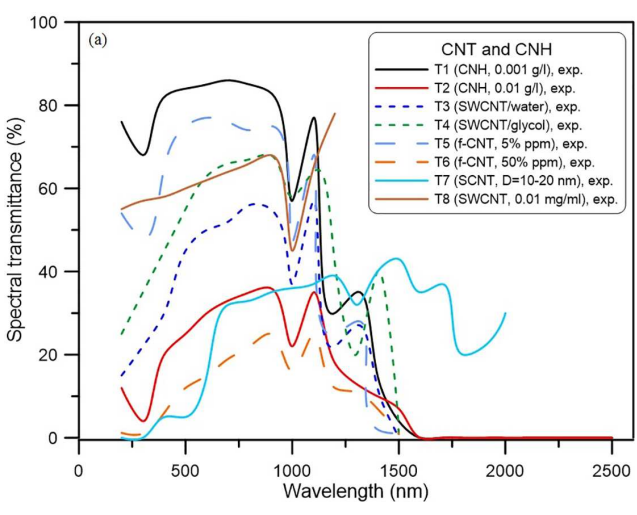

(a)

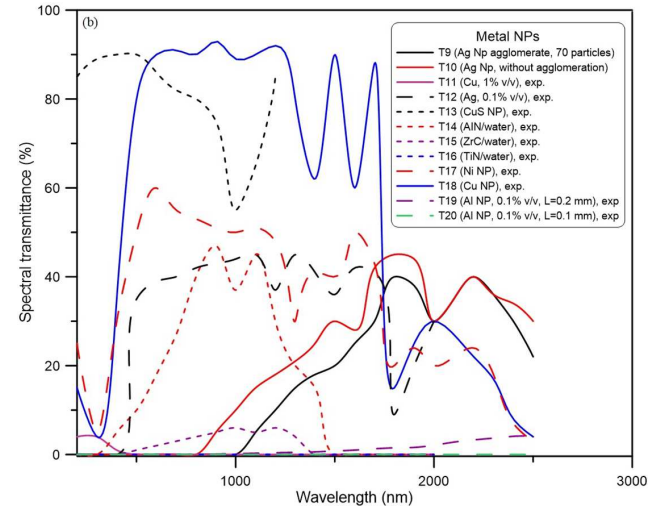

(b)

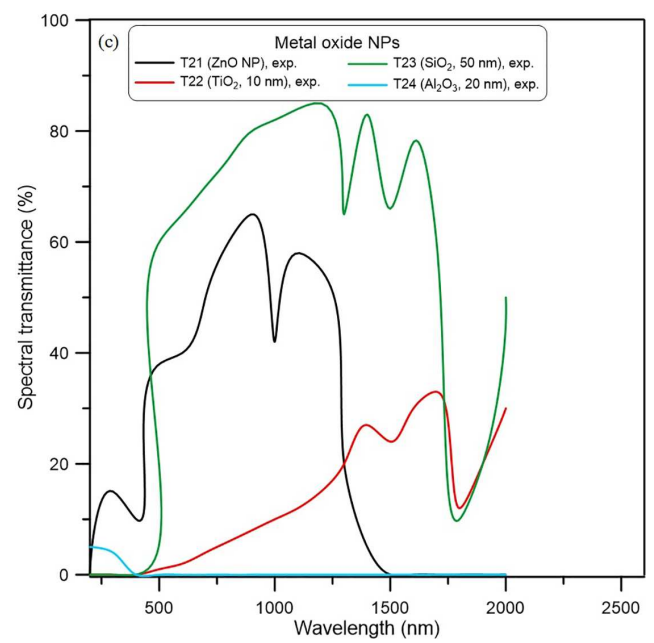

(c)

FIG. 12: Spectral transmittance profiles for different types of NPSs. (a) CNT and CNH NPs-based NpSs, T1 and T2 (Sani et al., 2010), T3 and T4 (Mercatelli et al., 2011), T5 and T6 (Karami et al., 2014), T7 (Luo et al., 2014), T8 (He et al., 2014). (b) Metal NPs-based NpSs, T9 and T10 (Du and Tang, 2015), T11 and T12 (Luo et al., 2014), T13 (He et al., 2014), T14-T16 (Zhu et al., 2013), T17 and T18 (Zhang et al., 2014), T19 and T20 (Saidur et al., 2012). (c) Metal oxide NPs-based NpSs, T21 (Zhu et al., 2013), T22-T24 (Luo et al., 2014).

a spectrophotometer is used (Zhu et al., 2013). Luo et al. studied the spectral transmittance of $\mathrm{TiO}_{2} /$ water (10 nm nominal size, $\left.1 \% v / v\right), \mathrm{SiO}_{2} /$ water (50 nm size, $\left.2 \% v / v\right), \mathrm{Al}_{2} \mathrm{O}_{3} /$ water $(20$ $\mathrm{nm}$ size, $2 \% v / v$ ). The medium was Texatherm oil. UV-vis-NIR spectrophotometer was used (Luo et al., 2014).

\section{RADIATIVE TRANSFER AND NANOPARTICLE SUSPENSIONS}

Nanoparticle suspensions bring promising heat transfer applications, which are of significant importance to a variety of industrial sectors such as transportation, power generation, 
micro-manufacturing, electronics, engines, thermal therapy, heating, cooling, ventilation, and air conditioning. Many of the reported anomalous enhancements in thermal conductivities in nanofluids were non-reproducible (Keblinski and Cahill, 2005; Raja et al., 2016; Gorji and Ranjbar, 2017; Rauf et al., 2019).

Solar energy utilization is an important area of research as solar radiation is a vast and renewable energy source. Unsustainable energy resources are unstable and expensive. Solar thermal collectors are the most important part in solar thermal systems; however, the effectiveness of these collectors is generally low because of the low radiative properties of the working fluids. The notion of direct absorption solar energy within a volume of fluid, which is called the direct absorption solar collectors, was initially introduced by Minardi and Chuang (1975). Nanoparticles are found to be effective radiation absorbers in low wavelength ranges, and they have unique thermal and optical properties, which are the basis of thermal applications (Green, 1982; Duffie and Beckman, 2013; Ali et al., 2018). A few researchers have attempted to investigate nanofluids for their suitability in improving the performance of the solar thermal collectors. The theoretical model that was introduced by Tyagi et al. was the very first model to quantitatively assess the effectiveness of a nanofluid-based direct absorption volumetric absorption solar collector (the working fluid describes a nanofluid-aluminum nanoparticles dispersed into water) (Tyagi et al., 2009). Referring to the progress in nanotechnology during the past years or so, the applications of nanoparticle suspensions in solar thermal collectors have been proposed because of their unique thermophysical and radiative properties (Green, 1982; Xuan and Li, 2000; Choi et al., 2004; Gorji and Ranjbar, 2017).

Researchers have also studied NPSs as potential volumetric absorbers as they promise better thermal efficiencies at high solar irradiance flux conditions. This may be attributed to the fact that as opposed to surface absorption-based receivers as here the sunlight directly interacts with the working fluid (nanofluid), which results in a more effective alteration of the solar irradiance to the thermal energy gain of the working fluid (Khullar et al., 2014; Khullar, 2016). In the backdrop of the ever-increasing energy demands and irreversible impact of burning fossil fuels to meet these demands, researchers around the globe are striving hard to improve existing solar thermal technologies (Veeraragavan et al., 2012; Phelan et al., 2013; Taylor et al., 2013; Hewakuruppu et al., 2015). Subsequently, Otanicar et al. (2010) and Taylor et al. (2011) have further refined the model to simulate actual nanofluid-sunlight interaction in volumetric absorption solar collectors. Furthermore, these refined models have been very well validated through the proof of the concept experiments done by these researchers. The aforementioned numerical, as well as experimental studies, clearly point out that under certain controlled conditions (and for the optimum selection of the nanoparticle material, shape, size, and concentration) an enhancement on the order of 5$10 \%$ could be achieved in the nanofluid-based volumetric collectors relative to the conventional solar collectors.

Various types of nanoparticles are being used in solar thermal collectors, and $\mathrm{TiO}_{2}$ and $\mathrm{Al}_{2} \mathrm{O}_{3}$ nanoparticles are extensively utilized where an enhancement in the thermal performance has been observed based on not only the thermophysical but also the radiative properties. Nanofluids have unique radiative properties, which play a significant role in photo-thermal energy conversion. Solar thermal collectors are the most important part in the solar thermal systems. However, the efficiency of conventional collectors (surface absorber collectors) is usually low due to the heat loss at the surface and the low radiative properties of the working fluids. This motivates the concept of using the nanofluids in the volumetric (direct absorption) solar collectors to enhance the thermal efficiency (Kim et al., 2016; Minkowycz et al., 2016). The investigations show that $\mathrm{TiO}_{2}$ and $\mathrm{Al}_{2} \mathrm{O}_{3}$ nanofluids have unique radiative properties, which play a significant role in the

Volume 27, Issue 3, 2020 
thermal performance of the photo-thermal energy conversion systems. The thermal efficiency of the proposed nanofluids in the concentrated solar collectors shows an enhancement (more than $10 \%$ ) compared to the conventional collectors under the same conditions (Muhammad et al., 2016; Jing and Song, 2017). The efficiency of the direct absorption solar collector is also examined, where the water-based aluminum nanoparticles are used as a working fluid, and it has been reported that an improvement in the collector efficiency by up to $10 \%$ was proposed (Tyagi et al., 2009). Nanosuspensions performance in solar collectors has been studied by Luo et al., and an improvement in the collector efficiency was observed up to approximately $2-25 \%$ as a result of the effect of adding nanoparticles (Luo et al., 2014). $\mathrm{TiO}_{2}$ nanoparticles have attracted attention not only because of their radiative and thermophysical properties, but also because they are very suitable for many applications and a wide range of applications such as energy production (solar thermal systems) (Cheng et al., 2005). Solar radiation is difficult to transmit through the $\mathrm{ZrC}$ and $\mathrm{TiO}_{2}$ nanofluid, while a remarkable amount of visible light can pass through the $\mathrm{SiO}_{2}$ nanofluid. A highest solar absorption is shown for the $\mathrm{ZrC}$ nanofluid among the studied nanofluids $(\mathrm{Mu}$ et al., 2009). A simulation model for a nanofluid solar collector based on the direct absorption collection concept for building utilization was developed by Luo et al. This model was developed through finding solutions to the radiative transfer equations of nanoparticulate media as well as by combining and convection heat transfer equations. The system efficiency/temperature distributions were examined by considering the nanoparticles absorption/scattering and the matrix absorption. The simulation results agreed well with the experimental data. The nanofluids improved the outlet temperature and efficiency by $30-100 \mathrm{~K}$ and $2-25 \%$, respectively (than the base fluid) (Luo et al., 2014).

A different experimental system was introduced by Zhang et al., which comprised a glass evacuated solar collector with an inserted heat pipe (Zhang et al., 2012). There were two different types of heat collector devices with a heat pipe using magnetic nanofluids and water as the working fluid, respectively, researched for different tilt angles, climate conditions, and total solar radiations. Various studies have shown that with the use of core-shell nanoparticles the efficiency of the direct absorption solar collector could be improved at very low volume fractions of the nanoparticles (Lv et al., 2013; Khullar, 2016; Khanafer and Vafai, 2018). It is envisaged that solar energy will be sustainably utilized in the near future instead of other alternative energy forms owing to its unlimited availability and desirable environmental and safety aspects. Because of the tremendous scientific and technological advances made during the last century and ongoing research and development, it is predicted that by 2100 , solar technologies will supply about $70 \%$ of world energy consumption (Council, 2013).

\section{CONCLUSIONS}

Nanoparticle suspensions have been used in different engineering applications, specifically in thermal applications. The interactions of electromagnetic radiation with the particles are of critical importance to a wide range of applications. Understanding this interaction and the phenomena that cover this field provides the information needed to develop performance and cost as well as optimizing systems and components, which include such applications. Promising applications can be achieved by manipulating the radiative properties of the NPSs with a trade-off between other properties (chemical and physical, etc.) of the medium and suspended particles. The goal is to enhance both the particles' radiative properties and their stability. Particle agglomeration cannot be avoided, which leads to unwanted effects. Then, the stability of these suspension types 
should be considered, and enhancing the stability (low agglomeration rate) of the suspended particles has a great impact on the optical and radiative properties of the NPSs which support their applications. The optical and radiative properties of the NPSs may change with the nanoparticle properties, and some of it affects individual particles and some affect the agglomerates. In addition, the characterization of the radiative properties of the particles and their agglomerates play a critical role in determining the radiative transfer of the surfaces in terms of the particle surface deposition and coating applications. The particle agglomerate size and surface-to-surface particle distance with their relationship to the wavelength are the most important parameters in the dependent and independent scattering.

The present study shows important concepts of particle agglomeration and their effects on the optical and radiative properties, the effects of different parameters have been included. The effects of particle stability, agglomeration, and sedimentation on the optical and radiative properties are significantly important in the radiative transfer in particulate suspensions. The study also outlines some simpler approaches to manipulate particle agglomeration, and consequently the optical/radiative properties of particles. This, in turn, affects the performance of NPSs for improved physical processes.

\section{ACKNOWLEDGMENT}

This work was supported by the Center for Energy, Environment, and Economy (CEEE) at Ozyegin University, Istanbul, Turkey.

\section{REFERENCES}

Afrand, M., Abedini, E., and Teimouri, H., Experimental Investigation and Simulation of Flow Boiling of Nanofluids in Different Flow Directions, Physica E, vol. 87, pp. 248-253, 2017.

Agrawal, B.M. and Mengüç, M.P., Forward and Inverse Analysis of Single and Multiple Scattering of Collimated Radiation in an Axisymmetric System, Int. J. Heat Mass Transf., vol. 34, no. 3, pp. 633-647, 1991.

Al-Gebory, L. and Mengüç, M.P., The Effect of $\mathrm{pH}$ on Particle Agglomeration and Optical Properties of Nanoparticle Suspensions, J. Quant. Spectrosc. Radiat. Transf., vol. 219, pp. 46-60, 2018.

Al-Gebory, L., Mengüç, M.P., Koşar, A., and Şendur, K., Effect of Electrostatic Stabilization on Thermal Radiation Transfer in Nanosuspensions: Photo-Thermal Energy Conversion Applications, Renewable Energy, vol. 119, pp. 625-640, 2018.

Ali, N., Teixeira, J.A., and Addali, A., A Review on Nanofluids: Fabrication, Stability, and Thermophysical Properties, J. Nanomater, vol. 2018, p. 6978130, 2018.

Allahyar, H., Hormozi, F., and ZareNezhad, B., Experimental Investigation on the Thermal Performance of a Coiled Heat Exchanger Using a New Hybrid Nanofluid, Exp. Therm. Fluid Sci., vol. 76, pp. 324-329, 2016.

Almohammed, N. and Breuer, M., Modeling and Simulation of Agglomeration in Turbulent Particle-Laden Flows: A Comparison between Energy-Based and Momentum-Based Agglomeration Models, Powder Technol., vol. 294, pp. 373-402, 2016.

Anushree, C. and Philip, J., Assessment of Long-Term Stability of Aqueous Nanofluids Using Different Experimental Techniques, J. Mol. Liq., vol. 222, pp. 350-358, 2016.

Asadi, A., Asadi, M., Rezaniakolaei, A., Rosendahl, L.A., Afrand, M., and Wongwises, S., Heat Transfer Efficiency of $\mathrm{Al}_{2} \mathrm{O}_{3}$-MWCNT Thermal Oil Hybrid Nanofluid as a Cooling Fluid in Thermal and Energy

Volume 27, Issue 3, 2020 
Management Applications: An Experimental and Theoretical Investigation, Int. J. Heat Mass Transf., vol. 117, pp. 474-486, 2018.

Aslan, M., Yamada, J., and Mengüç, M.P., Characterization of Individual Cotton Fibers via Light-Scattering Experiments, J. Thermophys. Heat Transf., vol. 17, no. 4, pp. 442-449, 2003.

Aslan, M.M., Crofcheck, C., Tao, D., and Mengüç, M.P., Evaluation of Micro-Bubble Size and Gas HoldUp in Two-Phase Gas-Liquid Columns via Scattered Light Measurements, J. Quant. Spectrosc. Radiat. Transf., vol. 101, no. 3, pp. 527-539, 2006a.

Aslan, M.M., Mengüç, M.P., Manickavasagam, S., and Saltiel, C., Size and Shape Prediction of Colloidal Metal Oxide MgBaFeO Particles from Light Scattering Measurements, J. Nanopart. Res., vol. 8, no. 6, pp. 981-994, 2006b.

Aziz, S., Experimental Investigation and Enhancement of Dispersion Stability of $\mathrm{TiO}_{2} / \mathrm{DI}$ Water Nanofluids for Thermal Energy Applications, PhD, USPCAS-E, NUST, 2018.

Azizi, Z., Alamdari, A., and Doroodmand, M.M., Highly Stable Copper/Carbon Dot Nanofluid: Preparation and Characterization, J. Therm. Anal. Calorim., vol. 133, no. 2, pp. 951-960, 2018.

Berne, B.J. and Pecora, R., Dynamic Light Scattering: With Applications to Chemistry, Biology, and Physics, New York: Dover Publications, pp. 120-175, 2000.

Bohren, C.F. and Huffman, D.R., Absorption and Scattering of Light by Small Particles, New York, NY: John Wiley and Sons, pp. 111-155, 2008.

Boisvert, J., Persello, J., Castaing, J., and Cabane, B., Dispersion of Alumina-Coated $\mathrm{TiO}_{2}$ Particles by Adsorption of Sodium Polyacrylate, Colloids Surf., A, vol. 178, nos. 1-3, pp. 187-198, 2001.

Chen, Q., Saltiel, C., Manickavasagam, S., Schadler, L.S., Siegel, R.W., and Yang, H., Aggregation Behavior of Single-Walled Carbon Nanotubes in Dilute Aqueous Suspension, J. Colloid Interf. Sci., vol. 280, no. 1, pp. 91-97, 2004.

Chen, W., Zou, C., Li, X., and Li, L., Experimental Investigation of SiC Nanofluids for Solar Distillation System: Stability, Optical Properties and Thermal Conductivity with Saline Water-Based Fluid, Int. J. Heat Mass Transf., vol. 107, pp. 264-270, 2017.

Cheng, P., Gu, M., and Jin, Y., Recent Progress in Titania Photocatalyst Operating under Visible Light, Prog. Chemis., vol. 17, no. 1, pp. 8-14, 2005.

Choi, S., Zhang, Z., and Keblinski, P., Nanofluids, Encyclopedia of Nanoscience and Nanotechnology, New York: HS Nalwa, pp. 757-773, 2004.

Choi, T.J., Jang, S.P., and Kedzierski, M., Effect of Surfactants on the Stability and Solar Thermal Absorption Characteristics of Water-Based Nanofluids with Multi-Walled Carbon Nanotubes, Int. J. Heat Mass Transf., vol. 122, pp. 483-490, 2018.

Colangelo, G., Favale, E., De Risi, A., and Laforgia, D., A New Solution for Reduced Sedimentation Flat Panel Solar Thermal Collector Using Nanofluids, Appl. Energy, vol. 111, pp. 80-93, 2013.

Cosgrove, T., Colloid Science: Principles, Methods and Applications, Hoboken, NJ: John Wiley and Sons, pp. 110-140, 2010.

Council, W.E., World Energy Resources, London, UK: World Energy Council, pp. 15-55, 2013.

Dai, X. and Haussener, S., Optical Characterization of Multi-Scale Morphologically Complex Heterogeneous Media-Application to Snow with Soot Impurities, J. Quant. Spectrosc. Radiat. Transf., vol. 206, pp. 378-391, 2018.

Dalkilic, A.S., Mahian, O., Kucukyildirim, B.O., Eker, A.A., Ozturk, T.H., Jumpholkul, C., and Wongwises, S., Experimental Study on the Stability and Viscosity for the Blends of Functionalized MWCNTs with Refrigeration Compressor Oils, Curr. Nanosci., vol. 14, no. 3, pp. 216-226, 2018.

Das, P.K., Mallik, A.K., Ganguly, R., and Santra, A.K., Stability and Thermophysical Measurements of $\mathrm{TiO}_{2}$ (Anatase) Nanofluids with Different Surfactants, J. Mol. Liq., vol. 254, pp. 98-107, 2018. 
Das, S.K., Choi, S.U., Yu, W., and Pradeep, T., Nanofluids: Science and Technology, Hoboken, NJ: John Wiley and Sons, pp. 120-170, 2007.

Daungthongsuk, W. and Wongwises, S., A Critical Review of Convective Heat Transfer of Nanofluids, Renewable Sustainable Energy Rev., vol. 11, no. 5, pp. 797-817, 2007.

Derjaguin, B., Theory of the Stability of Strongly Charged Lyophobic Sols and the Adhesion of Strongly Charged Particles in Solutions of Electrolytes, Acta Physicochim, vol. 14, pp. 633-662, 1941.

Devendiran, D.K. and Amirtham, V.A., A Review on Preparation, Characterization, Properties and Applications of Nanofluids, Renewable Sustainable Energy Rev., vol. 60, pp. 21-40, 2016.

DeVoe, H., Optical Properties of Molecular Aggregates I. Classical Model of Electronic Absorption and Refraction, J. Chem. Phys., vol. 41, no. 2, pp. 393-400, 1964.

Dhand, C., Dwivedi, N., Loh, X.J., Ying, A.N.J., Verma, N.K., Beuerman, R.W., Lakshminarayanan, R., and Ramakrishna, S., Methods and Strategies for the Synthesis of Diverse Nanoparticles and Their Applications: A Comprehensive Overview, RSC Adv., vol. 5, no. 127, pp. 105003-105037, 2015.

Dietz, P., The Effect of $\mathrm{TiO}_{2}$ Dispersion and Entrapped Air on the Opacity of Alkyd and Acrylic Emulsion Paints, Surf. Coat. Int., vol. 87, pp. 18-24, 2004.

Doicu, A. and Mishchenko, M.I., Radiative Transfer in a Discrete Random Medium Adjacent to a HalfSpace with a Rough Interface, J. Quant. Spectrosc. Radiat. Transf., vol. 218, pp. 194-202, 2018.

Dombrovsky, L., Dembele, S., Wen, J.X., and Sikic, I., Two-Step Iterative Method for Radiative Transfer Calculations in Axisymmetric Flames Containing Absorbing and Scattering Particles, in IHTC-16, 16th Int. Heat Transf. Conf., Beijing, China, 2018.

Dombrovsky, L.A. and Baillis, D., Thermal Radiation in Disperse Systems: An Engineering Approach, New York, NY: Begell House, pp. 15-35, 2010.

Draine, B.T. and Flatau, P.J., Discrete-Dipole Approximation for Scattering Calculations, J. Opt. Soc. Am. A., vol. 11, no. 4, pp. 1491-1499, 1994.

Drolen, B. and Tien, C., Independent and Dependent Scattering in Packed-Sphere Systems, J. Thermophys. Heat Transf., vol. 1, no. 1, pp. 63-68, 1987.

Du, M. and Tang, G., Optical Property of Nanofluids with Particle Agglomeration, Sol. Energy, vol. 122, pp. 864-872, 2015.

Duan, H. and Xuan, Y., Enhanced Optical Absorption of the Plasmonic Nanoshell Suspension based on the Solar Photocatalytic Hydrogen Production System, Appl. Energy, vol. 114, pp. 22-29, 2014.

Duffie, J.A. and Beckman, W.A., Solar Engineering of Thermal Processes, Hoboken, NJ: John Wiley and Sons, pp. 225-270, 2013.

Fazio, S., Guzman, J., Colomer, M., Salomoni, A., and Moreno, R., Colloidal Stability of Nanosized Titania Aqueous Suspensions, J. Eur. Ceram. Soc., vol. 28, no. 11, pp. 2171-2176, 2008.

Fedele, L., Colla, L., Bobbo, S., Barison, S., and Agresti, F., Experimental Stability Analysis of Different Water-Based Nanofluids, Nanoscale Res. Lett., vol. 6, no. 1, p. 300, 2011.

Flatau, P. and Draine, B., Fast near Field Calculations in the Discrete Dipole Approximation for Regular Rectilinear Grids, Opt. Express, vol. 20, no. 2, pp. 1247-1252, 2012.

Ghadimi, A. and Metselaar, I.H., The Influence of Surfactant and Ultrasonic Processing on Improvement of Stability, Thermal Conductivity and Viscosity of Titania Nanofluid, Exp. Therm. Fluid Sci., vol. 51, pp. 1-9, 2013.

Gibbs, J.W., On the Equilibrium of Heterogeneous Substances, Am. J. Sci., vol. 16, pp. 441-458, 1879.

Gorji, T.B. and Ranjbar, A., A Review on Optical Properties and Application of Nanofluids in Direct Absorption Solar Collectors (DASCs), Renewable Sustainable Energy Rev., vol. 72, pp. 10-32, 2017.

Gorji, T.B., Ranjbar, A., and Mirzababaei, S., Optical Properties of Carboxyl Functionalized Carbon Nanotube Aqueous Nanofluids as Direct Solar Thermal Energy Absorbers, Sol. Energy, vol. 119,

Volume 27, Issue 3, 2020 
pp. 332-342, 2015.

Green, M.A., Solar Cells: Operating Principles, Technology, and System Applications, Englewood Cliffs, NJ: Prentice-Hall, Inc., pp. 230-288, 1982.

Guo, Z., Heat Transfer Enhancement-A Brief Review of 2018 Literature, J. Enhanced Heat Transf., vol. 26, pp. 429-449, 2019.

Gupta, H., Agrawal, G., and Mathur, J., An Overview of Nanofluids: A New Media towards Green Environment, Int. J. Environ. Sci., vol. 3, no. 1, pp. 433-440, 2012.

Han, X., Guo, Y., Wang, Q., and Phelan, P., Optical Characterization and Durability of Immersion Cooling Liquids for High Concentration III-V Photovoltaic Systems, Sol. Energy Mater. Sol. Cells, vol. 174, pp. 124-131, 2018.

He, Q., Wang, S., Zeng, S., and Zheng, Z., Experimental Investigation on Photothermal Properties of Nanofluids for Direct Absorption Solar Thermal Energy Systems, Energy Convers. Manage., vol. 73, pp. 150-157, 2013.

He, Y., Jin, Y., Chen, H., Ding, Y., Cang, D., and Lu, H., Heat Transfer and Flow Behaviour of Aqueous Suspensions of $\mathrm{TiO}_{2}$ Nanoparticles (Nanofluids) Flowing Upward through a Vertical Pipe, Int. J. Heat Mass Transf., vol. 50, nos. 11-12, pp. 2272-2281, 2007.

He, Y., Vasiraju, S., and Que, L., Hybrid Nanomaterial-Based Nanofluids for Micropower Generation, RSC Adv., vol. 4, no. 5, pp. 2433-2439, 2014.

Hewakuruppu, Y.L., Taylor, R.A., Tyagi, H., Khullar, V., Otanicar, T., Coulombe, S., and Hordy, N., Limits of Selectivity of Direct Volumetric Solar Absorption, Sol. Energy, vol. 114, pp. 206-216, 2015.

Hoffman, C. and Driggers, R., Encyclopedia of Optical and Photonic Engineering, Emissivity-Lenses, New York, NY: Taylor and Francis/CRC Press, pp. 30-80, 2016.

Hordy, N., Rabilloud, D., Meunier, J.-L., and Coulombe, S., High Temperature and Long-Term Stability of Carbon Nanotube Nanofluids for Direct Absorption Solar Thermal Collectors, Sol. Energy, vol. 105, pp. 82-90, 2014.

Hossain, M.S., Saidur, R., Sabri, M.F.M., Said, Z., and Hassani, S., Spotlight on Available Optical Properties and Models of Nanofluids: A Review, Renewable Sustainable Energy Rev., vol. 43, pp. 750-762, 2015.

Howell, J.R., Mengüç, M.P., and Siegel, R., Thermal Radiation Heat Transfer, New York: Taylor and Francis/CRC Press, pp. 220-300, 2015.

Hulst, H.C., and Van de Hulst, H.C., Light Scattering by Small Particles, New York: Dover Publications, Inc., pp. 40-85, 1957.

Hunter, R.J., Foundations of Colloid Science, Great Clarendon, U.K.: Oxford University Press, pp. 25-50, 2001.

Hwang, Y., Lee, J., Lee, J., Jeong, Y., Cheong, S., Ahn, Y., and Kim, S.H., Production and Dispersion Stability of Nanoparticles in Nanofluids, Powder Technol., vol. 186, no. 2, pp. 145-153, 2008.

Ito, G., Mishchenko, M.I., and Glotch, T.D., Radiative-Transfer Modeling of Spectra of Planetary Regoliths Using Cluster-Based Dense Packing Modifications, J. Geophys. Res. Planets, vol. 123, no. 5, pp. 12031220, 2018.

Ivezić, Z. and Mengüç, M.P., An Investigation of Dependent/Independent Scattering Regimes Using a Discrete Dipole Approximation, Int. J. Heat Mass Transf., vol. 39, no. 4, pp. 811-822, 1996.

Ivezić, Ž., Mengüç, M.P., and Knauer, T.G., A Procedure to Determine the Onset of Soot Agglomeration from Multi-Wavelength Experiments, J. Quant. Spectrosc. Radiat. Transf., vol. 57, no. 6, pp. 859-865, 1997.

Jamil, M., Sidik, N.C., and Yazid, M.M., Thermal Performance of Thermosyphon Evacuated Tube Solar Collector Using $\mathrm{TiO}_{2} /$ Water Nanofluid, J. Adv. Res. Fluid Mech. Therm. Sci., vol. 20, no. 1, pp. 12-29, 
2016.

Jing, D. and Song, D., Optical Properties of Nanofluids Considering Particle Size Distribution: Experimental and Theoretical Investigations, Renewable Sustainable Energy Rev., vol. 78, pp. 452-465, 2017.

Kameya, Y. and Hanamura, K., Enhancement of Solar Radiation Absorption Using Nanoparticle Suspension, Sol. Energy, vol. 85, no. 2, pp. 299-307, 2011.

Karami, M., Bahabadi, M.A., Delfani, S., and Ghozatloo, A., A New Application of Carbon Nanotubes Nanofluid as Working Fluid of Low-Temperature Direct Absorption Solar Collector, Sol. Energy Mater. Sol. Cells, vol. 121, pp. 114-118, 2014.

Karimzadehkhouei, M., Ghorbani, M., Sezen, M., Şendur, K., Mengüç, M.P., Leblebici, Y., and Koşar, A., Increasing the Stability of Nanofluids with Cavitating Flows in Micro Orifices, Appl. Phys. Lett., vol. 109, no. 10, p. 104101, 2016.

Karimzadehkhouei, M., Shojaeian, M., Şendur, K., Mengüç, M.P., and Koşar, A., The Effect of Nanoparticle Type and Nanoparticle Mass Fraction on Heat Transfer Enhancement in Pool Boiling, Int. J. Heat Mass Transf., vol. 109, pp. 157-166, 2017.

Kawanami, T. and Sakurai, K., Cooling Performance of Room-Temperature Magnetic Refrigerator with Active Magnetic Regenerator (Numerical Analysis on Cooling Performance), Trans. JSME (B), vol. 73, no. 735, pp. 2323-2330, 2007.

Keblinski, P. and Cahill, D.G., Comment on "Model for Heat Conduction in Nanofluids," Phys. Rev. Lett., vol. 95, no. 20, p. 209401, 2005.

Keblinski, P., Eastman, J.A., and Cahill, D.G., Nanofluids for Thermal Transport, Mater. Today, vol. 8, no. 6, pp. 36-44, 2005.

Khanafer, K. and Vafai, K., A Review on the Applications of Nanofluids in Solar Energy Field, Renewable Energy, vol. 123, pp. 398-406, 2018.

Khullar, V., Heat Transfer Analysis and Optical Characterization of Nanoparticle Dispersion-Based Solar Thermal Systems, PhD, DSpace, 2016.

Khullar, V., Tyagi, H., Hordy, N., Otanicar, T.P., Hewakuruppu, Y., Modi, P., and Taylor, R.A., Harvesting Solar Thermal Energy through Nanofluid-Based Volumetric Absorption Systems, Int. J. Heat Mass Transf., vol. 77, pp. 377-384, 2014.

Kim, H., Ham, J., Park, C., and Cho, H., Theoretical Investigation of the Efficiency of a U-Tube Solar Collector Using Various Nanofluids, Energy, vol. 94, pp. 497-507, 2016.

Kiruba, R., Vinod, S., Zaibudeen, A., Solomon, R.V., and Philip, J., Stability and Rheological Properties of Hybrid $\gamma-\mathrm{Al}_{2} \mathrm{O}_{3}$ Nanofluids with Cationic Polyelectrolyte Additives, Colloids Surf., A, vol. 555, pp. 63$71,2018$.

Klusek, C., Manickavasagam, S., and Mengüç, M.P., Compendium of Scattering Matrix Element Profiles for Soot Agglomerates, J. Quant. Spectrosc. Radiat. Transf., vol. 79, pp. 839-859, 2003.

Konakanchi, H., Vajjha, R.S., Chukwu, G.A., and Das, D.K., Measurements of pH of Three Nanofluids and Development of New Correlations, Heat Transf. Eng., vol. 36, no. 1, pp. 81-90, 2015.

Kozan, M. and Mengüç, M.P., Exploration of Fractal Nature of $\mathrm{WO}_{3}$ Nanowire Aggregates, J. Quant. Spectrosc. Radiat. Transf., vol. 109, no. 2, pp. 327-336, 2008.

Kozan, M., Thangala, J., Bogale, R., Mengüç, M.P., and Sunkara, M.K., In-Situ Characterization of Dispersion Stability of $\mathrm{WO}_{3}$ Nanoparticles and Nanowires, J. Nanopart. Res., vol. 10, no. 4, pp. 599-612, 2008.

Kumar, S., Kothiyal, A.D., Bisht, M.S., and Kumar, A., Effect of Nanofluid Flow and Protrusion Ribs on Performance in Square Channels: An Experimental Investigation, J. Enhanced Heat Transf., vol. 26, no. 1, pp. 75-100, 2019.

Lattuada, M., Sandkühler, P., Wu, H., Sefcik, J., and Morbidelli, M., Aggregation Kinetics of Polymer

Volume 27, Issue 3, 2020 
Colloids in Reaction Limited Regime: Experiments and Simulations, Adv. Colloid Interf. Sci., vol. 103, no. 1, pp. 33-56, 2003.

Lax, M., Multiple Scattering of Waves. II. The Effective Field in Dense Systems, Phys. Rev., vol. 85, no. 4, p. $621,1952$.

Lee, B.J., Park, K., Walsh, T., and Xu, L., Radiative Heat Transfer Analysis in Plasmonic Nanofluids for Direct Solar Thermal Absorption, J. Sol. Energy Eng., vol. 134, no. 2, p. 021009-6, 2012.

Lee, S. and Jang, S.P., Extinction Coefficient of Aqueous Nanofluids Containing Multi-Walled Carbon Nanotubes, Int. J. Heat Mass Transf., vol. 67, pp. 930-935, 2013.

Lee, S., Choi, S., Li, S., and Eastman, J.A., Measuring Thermal Conductivity of Fluids Containing Oxide Nanoparticles, J. Heat Transf., vol. 121, no. 2, pp. 280-289, 1999.

Lee, S.H., Cho, E., Jeon, S.H., and Youn, J.R., Rheological and Electrical Properties of Polypropylene Composites Containing Functionalized Multi-Walled Carbon Nanotubes and Compatibilizers, Carbon, vol. 45, no. 14, pp. 2810-2822, 2007.

Liu, M., Lin, M.C., Huang, I., and Wang, C., Enhancement of Thermal Conductivity with Carbon Nanotube for Nanofluids, Int. Commun. Heat Mass Transf., vol. 32, no. 9, pp. 1202-1210, 2005.

Luo, Z., Wang, C., Wei, W., Xiao, G., and Ni, M., Performance Improvement of a Nanofluid Solar Collector based on Direct Absorption Collection (DAC) Concepts, Int. J. Heat Mass Transf., vol. 75, pp. 262-271, 2014.

Lv, W., Phelan, P.E., Swaminathan, R., Otanicar, T.P., and Taylor, R.A., Multifunctional Core-Shell Nanoparticle Suspensions for Efficient Absorption, J. Sol. Energy Eng., vol. 135, no. 2, p. 021004, 2013.

Mackowski, D.W. and Mishchenko, M.I., Calculation of the T Matrix and the Scattering Matrix for Ensembles of Spheres, J. Opt. Soc. Am. A, vol. 13, no. 11, pp. 2266-2278, 1996.

Madhesh, D. and Kalaiselvam, S., Energy Efficient Hybrid Nanofluids for Tubular Cooling Applications, App. Mech. Mater., vols. 592-593, p. 922, 2014.

Mahl, D., Diendorf, J., Meyer-Zaika, W., and Epple, M., Possibilities and Limitations of Different Analytical Methods for the Size Determination of a Bimodal Dispersion of Metallic Nanoparticles, Colloids Surf., A, vol. 377, nos. 1-3, pp. 386-392, 2011.

Manickavasagam, S. and Mengüç, M.P., Scattering Matrix Elements of Fractal-Like Soot Agglomerates, Appl. Opt., vol. 36, no. 6, pp. 1337-1351, 1997.

Maxwell, J.C., II. A Dynamical Theory of the Electromagnetic Field, Proc. Royal Society, London, vol. 13, pp. 531-536, 1864.

Menbari, A. and Alemrajabi, A.A., Analytical Modeling and Experimental Investigation on Optical Properties of New Class of Nanofluids $\left(\mathrm{Al}_{2} \mathrm{O}_{3}-\mathrm{CuO}\right.$ Binary Nanofluids) for Direct Absorption Solar Thermal Energy, Opt. Mater., vol. 52, pp. 116-125, 2016.

Mengiic, M. and Viskanta, R., An Assessment of Spectral Radiative Heat Transfer Predictions for a Pulverized Coal-Fired Furnace, 8th Int. Heat Transfer Conference, Begell House Inc., pp. 815-820, 1986.

Mengüç, M. and Manickavasagam, S., Characterization of Size and Structure of Agglomerates and Inhomogeneous Particles via Polarized Light, Int. J. Eng. Sci., vol. 36, nos. 12-14, pp. 1569-1593, 1998.

Mengüç, M.P., Characterization of Fine Particles via Elliptically-Polarized Light Scattering, in Purdue Heat Transfer Celebration, West Lafayette, IN, 2003.

Mercatelli, L., Sani, E., Zaccanti, G., Martelli, F., Di Ninni, P., Barison, S., Pagura, C., Agresti, F., and Jafrancesco, D., Absorption and Scattering Properties of Carbon Nanohorn-Based Nanofluids for Direct Sunlight Absorbers, Nanoscale Res. Lett., vol. 6, no. 1, pp. 1-9, 2011.

Mewis, J. and Wagner, N.J., Colloidal Suspension Rheology, Cambridge, UK: Cambridge University Press, 2012. 
Mie, G., Beiträge zur Optik Trüber Medien, Speziell Kolloidaler Metallösungen, Annalen Physik, vol. 330, no. 3, pp. 377-445, 1908 .

Minardi, J.E. and Chuang, H.N., Performance of a "Black" Liquid Flat-Plate Solar Collector, Sol. Energy, vol. 17, no. 3, pp. 179-183, 1975.

Minkowycz, W., Sparrow, E.M., and Abraham, J.P., Nanoparticle Heat Ttransfer and Fluid Flow, New York: Taylor and Francis/CRC Press, pp. 220-300, 2015.

Mishchenko, M.I., Electromagnetic Scattering by Particles and Particle Groups: An Introduction: Cambridge, UK: Cambridge University Press, pp. 40-80, 2014.

Mishchenko, M.I., Electromagnetic Scattering and Emission by a Fixed Multi-Particle Object in Local Thermal Equilibrium: General Formalism, J. Quant. Spectrosc. Radiat. Transf., vol. 200, pp. 137-145, 2017.

Mishchenko, M.I. and Dlugach, J.M., Scattering and Extinction by Spherical Particles Immersed in an Absorbing Host Medium, J. Quant. Spectrosc. Radiat. Transf., vol. 211, pp. 179-187, 2018.

Mishchenko, M.I. and Dlugach, J.M., Multiple Scattering of Polarized Light by Particles in an Absorbing Medium, Appl. Opt., vol. 58, no. 18, pp. 4871-4877, 2019.

Mishchenko, M.I., Hovenier, J.W., and Travis, L.D., Light Scattering by Nonspherical Particles: Theory, Measurements, and Applications, London: Academic Press, pp. 120-200, 1999.

Mishchenko, M.I., Travis, L.D., and Lacis, A.A., Scattering, Absorption, and Emission of Light by Small Particles, Cambridge, UK: Cambridge University Press, 2002.

Mishchenko, M.I., Travis, L.D., and Mackowski, D.W., T-Matrix Computations of Light Scattering by Nonspherical Particles: A Review, J. Quant. Spectrosc. Radiat. Transf., vol. 55, no. 5, pp. 535-575, 1996.

Mishchenko, M.I., Videen, G., and Yang, P., Extinction by a Homogeneous Spherical Particle in an Absorbing Medium, Opt. Lett., vol. 42, no. 23, pp. 4873-4876, 2017.

Mishchenko, M.I. and Yurkin, M.A., On the Concept of Random Orientation in Far-Field Electromagnetic Scattering by Nonspherical Particles, Opt. Lett., vol. 42, no. 3, pp. 494-497, 2017.

Modest, M.F., Radiative Heat Transfer, New York, NY: Academic Press, pp. 200-250, 2013.

Mu, L., Zhu, Q., and Si, L., Radiative Properties of Nanofluids and Performance of a Direct Solar Absorber Using Nanofluids, ASME 2009, 2nd Int. Conf. on Micro/Nanoscale Heat and Mass Transfer, 2009.

Muhammad, M.J., Muhammad, I.A., Sidik, N.A.C., Yazid, M.N.A.W.M., Mamat, R., and Najafi, G., The Use of Nanofluids for Enhancing the Thermal Performance of Stationary Solar Collectors: A Review, Renewable Sustainable Energy Rev., vol. 63, pp. 226-236, 2016.

Muthusamy, Y., Kadirgama, K., Rahman, M., Ramasamy, D., and Sharma, K., Wear Analysis when Machining AISI 304 with Ethylene Glycol/TiO 2 Nanoparticle-Based Coolant, Int. J. Adv. Manuf. Technol., vol. 82, nos. 1-4, pp. 327-340, 2016.

Nayak, S.K. and Mishra, P.C., Enhanced Heat Transfer from Hot Surface by Nanofluid based Ultrafast Cooling: An Experimental Investigation, J. Enhanced. Heat Transf., vol. 26, no. 4, pp. 415-428, 2019.

Ortega, M.A., Rodriguez, L., Castillo, J., Fernández, A., and Echevarria, L., Thermo-Optical Properties of Nanofluids, AIP Conf. Proc., vol. 992, no. 1, 2008.

Otanicar, T., Taylor, R.A., Phelan, P.E., and Prasher, R., Impact of Size and Scattering Mode on the Optimal Solar Absorbing Nanofluid, ASME 2009, 3rd Int. Conf. on Energy Sustainability, vol. 1, pp. 791-796, 2009.

Otanicar, T.P., Phelan, P.E., Prasher, R.S., Rosengarten, G., and Taylor, R.A., Nanofluid-Based Direct Absorption Solar Collector, J. Renewable Sustainable Energy, vol. 2, no. 3, p. 033102, 2010.

Phelan, P., Otanicar, T., Taylor, R., and Tyagi, H., Trends and Opportunities in Direct-Absorption Solar Thermal Collectors, J. Therm. Sci. Eng. Appl., vol. 5, no. 2, p. 021003, 2013.

Volume 27, Issue 3, 2020 
Powers, K.W., Brown, S.C., Krishna, V.B., Wasdo, S.C., Moudgil, B.M., and Roberts, S.M., Research Strategies for Safety Evaluation of Nanomaterials. Part VI. Characterization of Nanoscale Particles for Toxicological Evaluation, Toxicol. Sci., vol. 90, no. 2, pp. 296-303, 2006.

Prasher, R., Phelan, P.E., and Bhattacharya, P., Effect of Aggregation Kinetics on the Thermal Conductivity of Nanoscale Colloidal Solutions (Nanofluid), Nano Lett., vol. 6, no. 7, pp. 1529-1534, 2006.

Prasher, R.S. and Phelan, P.E., Modeling of Radiative and Optical Behavior of Nanofluids based on Multiple and Dependent Scattering Theories, ASME 2005, Int. Mech. Eng. Cong. and Expo., pp. 739-743, 2005.

Purcell, E.M. and Pennypacker, C.R., Scattering and Absorption of Light by Nonspherical Dielectric Grains, Astrophys. J., vol. 186, pp. 705-714, 1973.

Raja, M., Vijayan, R., Dineshkumar, P., and Venkatesan, M., Review on Nanofluids Characterization, Heat Transfer Characteristics and Applications, Renewable Sustainable Energy Rev., vol. 64, pp. 163-173, 2016.

Randrianalisoa, J. and Baillis, D., Radiative Transfer in Dispersed Media: Comparison between Homogeneous Phase and Multiphase Approaches, J. Heat Transf., vol. 132, no. 2, pp. 163-173, 2010.

Rativa, D. and Gómez-Malagón, L.A., Solar Radiation Absorption of Nanofluids Containing Metallic Nanoellipsoids, Sol. Energy, vol. 118, pp. 419-425, 2015.

Rauf, A., Abbas, Z., and Shehzad, S.A., Chemically Reactive Hydromagnetic Flow over a Stretchable Oscillatory Rotating Disk with Thermal Radiation and Heat Source/Sink: A Numerical Study, Heat Transf. Res., vol. 50, no. 15, pp. 1495-1512, 2019.

Rayleigh, L., On the Light from the Sky: Its Polarization and Colour, Philos. Mag., vol. 41, p. 274, 1871.

Russel, W.B., Saville, D.A., and Schowalter, W.R., Colloidal Dispersions, New York: Cambridge University Press, pp. 55-150, 1989.

Safaei-Naeini, Y., Aminzare, M., Golestani-Fard, F., Khorasanizadeh, F., and Salahi, E., Suspension Stability of Titania Nanoparticles Studied by UV-VIS Spectroscopy Method, Iran. J. Mater. Sci. Eng., vol. 9, no. 1, pp. 62-68, 2012.

Saghrouni, Z., Baillis, D., Naouar, N., Blal, N., and Jemni, A., Thermal Properties of New Insulating Juncus Maritimus Fibrous Mortar Composites/Experimental Results and Analytical Laws, Appl. Sci., vol. 9, no. 5, p. 981, 2019.

Said, Z., Saidur, R., and Rahim, N., Optical Properties of Metal Oxides based Nanofluids, Int. Commun. Heat Mass Transf., vol. 59, pp. 46-54, 2014.

Said, Z., Sajid, M., Alim, M., Saidur, R., and Rahim, N., Experimental Investigation of the Thermophysical Properties of $\mathrm{Al}_{2} \mathrm{O}_{3}$-Nanofluid and its Effect on a Flat Plate Solar Collector, Int. Commun. Heat Mass Transf., vol. 48, pp. 99-107, 2013.

Said, Z., Sajid, M., Saidur, R., Mahdiraji, G., and Rahim, N., Evaluating the Optical Properties of $\mathrm{TiO}_{2}$ Nanofluid for a Direct Absorption Solar Collector, Numer. Heat Transf., Part A, vol. 67, no. 9, pp. 10101027, 2015.

Saidur, R., Meng, T., Said, Z., Hasanuzzaman, M., and Kamyar, A., Evaluation of the Effect of NanofluidBased Absorbers on Direct Solar Collector, Int. J. Heat Mass Transf., vol. 55, nos. 21-22, pp. 5899-5907, 2012.

Sajid, M., Said, Z., Saidur, R., Adikan, F., Sabri, M., and Rahim, N., A Time Variant Investigation on Optical Properties of Water based $\mathrm{Al}_{2} \mathrm{O}_{3}$ Nanofluid, Int. Commun. Heat Mass Transf., vol. 50, pp. 108116, 2014.

Sajid, M., Said, Z., Saidur, R., Sabri, M., and Faizul, M., Applicability of Alumina Nanofluid in Direct Absorption Solar Collectors, Appl. Mech. Mater, vol. 699, pp. 366-371, 2015.

Saltiel, C., Chen, Q., Manickavasagam, S., Schadler, L., Siegel, R., and Mengüç, M.P., Identification of the Dispersion Behavior of Surface Treated Nanoscale Powders, J. Nanopart. Res., vol. 6, no. 1, pp. 35-46, 2004. 
Saltiel, C., Manickavasagam, S., Mengüc, M.P., and Andrews, R., Light-Scattering and Dispersion Behavior of Multiwalled Carbon Nanotubes, J. Opt. Soc. Am. A, vol. 22, no. 8, pp. 1546-1554, 2005.

Sani, E., Barison, S., Pagura, C., Mercatelli, L., Sansoni, P., Fontani, D., Jafrancesco D., and Francini, F., Carbon Nanohorns-Based Nanofluids as Direct Sunlight Absorbers, Opt. Express, vol. 18, no. 5, pp. 5179-5187, 2010.

Sarkar, J., Ghosh, P., and Adil, A., A Review on Hybrid Nanofluids: Recent Research, Development and Applications, Renewable Sustainable Energy Rev., vol. 43, pp. 164-177, 2015.

Selvakumar, P. and Suresh, S., Use of $\mathrm{Al}_{2} \mathrm{O}_{3}-\mathrm{Cu} /$ Water Hybrid Nanofluid in an Electronic Heat Sink, IEEE Trans. Compon. Packag. Manuf. Technol., vol. 2, no. 10, pp. 1600-1607, 2012.

Sen, S., Govindarajan, V., Pelliccione, C.J., Wang, J., Miller, D.J., and Timofeeva, E.V., Surface Modification Approach to $\mathrm{TiO}_{2}$ Nanofluids with High Particle Concentration, Low Viscosity, and Electrochemical Activity, ACS Appl. Mater. Interf., vol. 7, no. 37, pp. 20538-20547, 2015.

Shende, R. and Sundara, R., Nitrogen Doped Hybrid Carbon based Composite Dispersed Nanofluids as Working Fluid for Low-Temperature Direct Absorption Solar Collectors, Sol. Energy Mater. Sol. Cells, vol. 140, pp. 9-16, 2015.

Simakov, S.A. and Tsur, Y., Surface Stabilization of Nano-Sized Titanium Dioxide: Improving the Colloidal Stability and the Sintering Morphology, J. Nanopart. Res., vol. 9, no. 3, pp. 403-417, 2007.

Singham, S.B. and Bohren, C.F., Light Scattering by an Arbitrary Particle: The Scattering-Order Formulation of the Coupled-Dipole Method, J. Opt. Soc. Am. A, vol. 5, no. 11, pp. 1867-1872, 1988.

Sinz, C., Woei, H., Khalis, M., and Abbas, S., Numerical Study on Turbulent Force Convective Heat Transfer of Hybrid Nanofluid, Ag/HEG in a Circular Channel with Constant Heat Flux, J. Adv. Res. Fluid Mech. Therm. Sci., vol. 24, no. 1, pp. 1-11, 2016.

Song, D., Hatami, M., Wang, Y., Jing, D., and Yang, Y., Prediction of Hydrodynamic and Optical Properties of $\mathrm{TiO}_{2} /$ Water Suspension Considering Particle Size Distribution, Int. J. Heat Mass Transf., vol. 92, pp. 864-876, 2016.

Sundar, L.S., Sharma, K., Singh, M.K., and Sousa, A., Hybrid Nanofluids Preparation, Thermal Properties, Heat Transfer and Friction Factor-A Review, Renewable Sustainable Energy Rev., vol. 68, pp. 185-198, 2017.

Swamy, J., Crofcheck, C., and Mengüç, M.P., Time Dependent Scattering Properties of Slow Decaying Liquid Foams, Colloids Surf., A, vol. 338, nos. 1-3, pp. 80-86, 2009.

Swinehart, D., The Beer-Lambert Law, J. Chem. Educ., vol. 39, no. 7, p. 333, 1962.

Tadros, T.F., Dispersion of Powders in Liquids and Stabilization of Suspensions, New York, NY: John Wiley and Sons, pp. 60-150, 2012.

Tan, J., Xie, Y., Wang, F., Jing, L., and Ma, L., Investigation of Optical Properties and Radiative Transfer of $\mathrm{TiO}_{2}$ Nanofluids with the Consideration of Scattering Effects, Int. J. Heat Mass Transf., vol. 115, pp. 1103-1112, 2017.

Taylor, R., Coulombe, S., Otanicar, T., Phelan, P., Gunawan, A., Lv, W., Rosengarten, G., Prasher, R., and Tyagi, H., Small Particles, Big Impacts: A Review of the Diverse Applications of Nanofluids, J. Appl. Phys., vol. 113, no. 1, 2013.

Taylor, R.A., Phelan, P.E., Otanicar, T.P., Adrian, R., and Prasher, R., Nanofluid Optical Property Characterization: Towards Efficient Direct Absorption Solar Collectors, Nanoscale Res. Lett., vol. 6, no. 1, p. 225, 2011.

Trisaksri, V. and Wongwises, S., Critical Review of Heat Transfer Characteristics of Nanofluids, Renewable Sustainable Energy Rev., vol. 11, no. 3, pp. 512-523, 2007.

Turcu, R., Nan, A., Craciunescu, I., Karsten, S., Pana, O., Bratu, I., Bica, D., Vekas, L. Chauvet, O., Eberbeck, D., and Ahlers, H., Functionalized Nanostructures with Magnetic Core and Pyrrole Copolymers Shell, J. Nanostruct. Polymers Nanocompos., vol. 3, no. 2, p. 55, 2007.

Volume 27, Issue 3, 2020 
Tyagi, H., Phelan, P., and Prasher, R., Predicted Efficiency of a Low-Temperature Nanofluid-Based Direct Absorption Solar Collector, J. Sol. Energy Eng., vol. 131, no. 4, p. 041004, 2009.

Vakili, M., Mohebbi, A., and Hashemipour, H., Experimental Study on Convective Heat Transfer of $\mathrm{TiO}_{2}$ Nanofluids, J. Heat Mass Transf., vol. 49, no. 8, pp. 1159-1165, 2013.

Veeraragavan, A., Lenert, A., Yilbas, B., Al-Dini, S., and Wang, E.N., Analytical Model for the Design of Volumetric Solar Flow Receivers, Int. J. Heat Mass Transf., vol. 55, no. 4, pp. 556-564, 2012.

Verwey, E., Theory of the Stability of Lyophobic Colloids, J. Phys. Chem., vol. 51, no. 3, pp. 631-636, 1947.

Viskanta, R. and Mengüç, M.P., Radiative Transfer in Dispersed Media, Appl. Mech. Rev., vol. 42, no. 9 , pp. 241-259, 1989.

Wamkam, C.T., Opoku, M.K., Hong, H., and Smith, P., Effects of pH on Heat Transfer Nanofluids Containing $\mathrm{ZrO}_{2}$ and $\mathrm{TiO}_{2}$ Nanoparticles, J. Appl. Phys., vol. 109, no. 2, p. 024305, 2011.

Wang, B.-X., Zhou, L.-P., and Peng, X.-F., A Fractal Model for Predicting the Effective Thermal Conductivity of Liquid with Suspension of Nanoparticles, Int. J. Heat Mass Transf., vol. 46, no. 14, pp. 2665-2672, 2003.

Wang, B. and Zhao, C., Effect of Dependent Scattering on Light Absorption in Highly Scattering Random Media, Int. J. Heat Mass Transf., vol. 125, pp. 1069-1078, 2018.

Wang, X.-Q. and Mujumdar, A.S., A Review on Nanofluids-Part I: Theoretical and Numerical Investigations, Braz. J. Chem. Eng., vol. 25, no. 4, pp. 613-630, 2008.

Waterman, P., Matrix Formulation of Electromagnetic Scattering, Proc. IEEE, vol. 53, no. 8, pp. 805-812, 1965.

Wei, W., Fedorov, A.G., Luo, Z., and Ni, M., Radiative Properties of Dense Nanofluids, Appl. Opt., vol. 51, no. 25, pp. 6159-6171, 2012.

Wen, D., Lin, G., Vafaei, S., and Zhang, K., Review of Nanofluids for Heat Transfer Applications, Particuology, vol. 7, no. 2, pp. 141-150, 2009.

Widegren, J. and Bergström, L., Electrostatic Stabilization of Ultrafine Titania in Ethanol, J. Am. Ceram. Soc., vol. 85, no. 3, pp. 523-528, 2002.

Williams, D.N., Ehrman, S.H., and Holoman, T.R.P., Evaluation of the Microbial Growth Response to Inorganic Nanoparticles, J. Nanobiotechnol., vol. 4, no. 1, p. 3, 2006.

Wu, Y., Yang, W., Wang, C., Hu, J., and Fu, S., Chitosan Nanoparticles as a Novel Delivery System for Ammonium Glycyrrhizinate, Int. J. Pharm., vol. 295, nos. 1-2, pp. 235-245, 2005.

Xian-Ju, W., Hai, L., Xin-Fang, L., Zhou-Fei, W., and Fang, L., Stability of $\mathrm{TiO}_{2}$ and $\mathrm{Al}_{2} \mathrm{O}_{3}$ Nanofluids, Chin. Phys. Lett., vol. 28, no. 8, p. 086601, 2011.

Xiao, B., Chen, H., Xiao, S., and Cai, J., Research on Relative Permeability of Nanofibers with Capillary Pressure Effect by Means of Fractal-Monte Carlo Technique, J. Nanosci. Nanotechnol., vol. 17, no. 9, pp. 6811-6817, 2017.

Xie, H., Fujii, M., and Zhang, X., Effect of Interfacial Nanolayer on the Effective Thermal Conductivity of Nanoparticle-Fluid Mixture, Int. J. Heat Mass Transf., vol. 48, no. 14, pp. 2926-2932, 2005.

Xuan, Y. and Li, Q., Heat Transfer Enhancement of Nanofluids, Int. J. Heat Fluid Flow, vol. 21, no. 1, pp. 58-64, 2000.

Yang, L. and Du, K., A Comprehensive Review on Heat Transfer Characteristics of $\mathrm{TiO}_{2}$ Nanofluids, Int. J. Heat Mass Transf., vol. 108, pp. 11-31, 2017.

Yang, L., Du, K., and Zhang, X., A Theoretical Investigation of Thermal Conductivity of Nanofluids with Particles in Cylindrical Shape by Anisotropy Analysis, Powder Technol., vol. 314, pp. 328-338, 2017.

Yousefi, T., Shojaeizadeh, E., Veysi, F., and Zinadini, S., An Experimental Investigation on the Effect of $\mathrm{pH}$ Variation of MWCNT- $\mathrm{H}_{2} \mathrm{O}$ Nanofluid on the Efficiency of a Flat-Plate Solar Collector, Sol. Energy, 
vol. 86, no. 2, pp. 771-779, 2012.

Yousif, M.A., Ismael, H.F., Abbas, T., and Ellahi, R., Numerical Study of Momentum and Heat Transfer of MHD Carreau Nanofluid over an Exponentially Stretched Plate with Internal Heat Source/Sink and Radiation, Heat Transf. Res., vol. 50, no. 7, pp. 649-658, 2019.

Yurkin, M.A., and Hoekstra, A.G., The Discrete Dipole Approximation: An Overview and Recent Developments, J. Quant. Spectrosc. Radiat. Transf., vol. 106, nos. 1-3, pp. 558-589, 2007.

Yurkin, M.A., Maltsev, V.P., and Hoekstra, A.G., The Discrete Dipole Approximation for Simulation of Light Scattering by Particles Much Larger than the Wavelength, J. Quant. Spectrosc. Radiat. Transf., vol. 106, nos. 1-3, pp. 546-557, 2007.

Zhang, L., Liu, J., He, G., Ye, Z., Fang, X., and Zhang, Z., Radiative Properties of Ionic Liquid-Based Nanofluids for Medium-to-High-Temperature Direct Absorption Solar Collectors, Sol. Energy Mater. Sol. Cells, vol. 130, pp. 521-528, 2014.

Zhang, Y.F., Han, K., Xu, B.L., and Chang, Z.R., Experimental Study on Heat Transfer Performance of the Solar Collector with an Inserted Heat Pipe Using Magnetic Nanofluids as the Working Fluid, Appl. Mech. Mater, vols. 209-211, pp. 279-283, 2012.

Zhu, D., Li, X., Wang, N., Wang, X., Gao, J., and Li, H., Dispersion Behavior and Thermal Conductivity Characteristics of $\mathrm{Al}_{2} \mathrm{O}_{3}-\mathrm{H}_{2} \mathrm{O}$ Nanofluids, Curr. Appl. Phys., vol. 9, no. 1, pp. 131-139, 2009.

Zhu, Q., Cui, Y., Mu, L., and Tang, L., Characterization of Thermal Radiative Properties of Nanofluids for Selective Absorption of Solar Radiation, Int. J. Thermophys., vol. 34, no. 12, pp. 2307-2321, 2013.

Zubko, E., Shkuratov, Y., and Videen, G., Effect of Morphology on Light Scattering by Agglomerates, $J$. Quant. Spectrosc. Radiat. Transf., vol. 150, pp. 42-54, 2015.

Volume 27, Issue 3, 2020 